\title{
Inspired by art:
}

\section{Higher aesthetic appeal elicits increased felt inspiration in a creative writing task}

\author{
Dominik Welke ${ }^{\mathrm{a}}$, Isaac Purton ${ }^{\mathrm{b}}$, and Edward A. Vessel ${ }^{\mathrm{a}}$ \\ ${ }^{a}$ Max-Planck-Institute for Empirical Aesthetics, Department of Neuroscience, \\ Grueneburgweg 14, D-60322 Frankfurt a.M., Germany

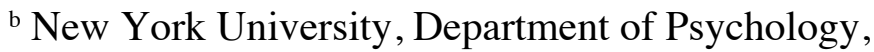 \\ 6 Washington Place, New York, NY 10003 USA
}

Dominik Welke,dominik.welke@ae.mpg.de, (D https://orcid.org/0000-0002-5529-1998 Isaac Purton, ipurton@gmail.com

Edward A. Vessel, ed.vessel@ae.mpg.de, (D https://orcid.org/0000-0001-8733-1731

Author Statement (CRediT) - DW: Conceptualization, Investigation, Formal Analysis, Software, Data curation, Project administration, Visualization, Writing - Original Draft, Writing - Review \& Editing; IP: Conceptualization, Methodology, Investigation, Formal Analysis, Software, Writing - Original Draft, Writing - Review \& Editing; EV:

Conceptualization, Methodology, Software, Writing - Original Draft, Writing - Review \& Editing, Resources, Supervision, Project administration, Funding acquisition

Correspondence concerning this article should be addressed to Edward A. Vessel, Max-Planck-Institute for Empirical Aesthetics, Grueneburgweg 14, D-60322 Frankfurt a.M., Germany.Email: ed.vessel@ae.mpg.de

(C) 2021, American Psychological Association. This paper is not the copy of record and may not exactly replicate the final, authoritative version of the article. Please do not copy or cite without authors' permission. The final article will be available, upon publication, via its DOI: 10.1037/aca0000393 


\begin{abstract}
Moments of creative inspiration - an evoked state of motivation for creative activity - form a distinct step in creative processes. We hypothesize that the psychological state of being creatively inspired is similar to the state of being aesthetically moved, and that aesthetically moving experiences can serve as prompts for creative inspiration. We tested this hypothesis in a creative writing task: In Experiment 1,25 participants from the general public were asked to write 12 short creative vignettes (repeated measures) in response to either "aesthetic" prompts (visual artworks they had rated highly aesthetically moving in a preceding task) or "nonaesthetic" prompts (triads of unrelated words). People then rated how inspired they had felt during idea generation. Ratings of inspiration were significantly higher for aesthetic vs. nonaesthetic prompts. In Experiment 2, 34 participants performed the same task with prompts consisting of highly-moving artworks, non-moving artworks (rated in a preceding task), or novel artworks not seen in advance. While pre-exposure to the stimuli had no significant effect, felt inspiration was significantly higher for moving vs. non-moving prompts, and posttest aesthetic ratings of novel artworks were positively correlated with felt inspiration. Furthermore, inspiration ratings correlated positively with the amount of produced text. Being aesthetically moved increases the likelihood of creative inspiration, a finding with implications both for the study of creative and aesthetic processes and also for the potential role of the arts in educational settings.
\end{abstract}

Keywords: felt inspiration, aesthetic appreciation, creativity, visual art, creative writing 


\section{Introduction}

While the concept of an artistic muse dates to antiquity, modern cognitive psychology has much to learn about the mental processes that support the generation of original or creative ideas. Given the high cultural and economic relevance attached to creative ideas and achievements, it is no surprise that the past two decades have seen intense work on the psychological mechanisms supporting creative thought at a number of levels of analysis, from inter-personal traits down to moment-to-moment mental operations (for reviews see Batey \& Furnham, 2006; Dietrich \& Kanso, 2010; Scott, Leritz, \& Mumford, 2004a).

At the level of character traits, it is known that individual abilities, such as the capacity for divergent and convergent thinking (introduced by Guilford, 1950), general intelligence, and openness (or open-mindedness; Jauk, Benedek, \& Neubauer, 2014) are all linked to creativity (see Batey \& Furnham, 2006 for a review). Trait-level creativity is often assessed by standardized tools like the Alternative-Uses Task (AUT; Guilford, Christensen, Merrifield, \& Wilson, 1978) but also via quantitative or qualitative ratings of the creativity of outputs, and recent efforts have linked trait-level creativity to neural systems such as the dopaminergic system (Boot, Nevicka, \& Baas, 2017), the default-mode network (Boccia, Piccardi, Palermo, Nori, \& Palmiero, 2015; Gonen-Yaacovi et al., 2013), the executivecontrol network (Santarnecchi, Emmendorfer, \& Pascual-Leone, 2017), and to functional connectivity across brain systems (Beaty et al., 2018); see Jauk (2019) for a recent review. Beyond inter-personal differences in creative ability, it is also the case that a persons' creativity can vary - not only over one's lifetime (Baer, 1994; Scott, Leritz, \& Mumford, 2004b; Torrance, 1968) but also over the course of days, hours, or even minutes (Amabile, 1985; Zenasni \& Lubart, 2002). This likely holds true for all individuals, not just those that exhibit high trait-level creativity - nearly everyone has the capacity to engage in some form of creative thought. Influential models of creativity take this into account, recognizing that 
while culturally relevant creative achievements (Carson, Peterson, \& Higgins, 2005) are only produced by a small subset of the population (Eysenck, 1995), everyday creative activity is rather commonplace and not confined to a subset of the population (Jauk et al., 2014). Rather, the capacity for creative and artistic activity is likely a core skill of humankind that plays a role in learning, personal development and professional activities (see e.g. the 4C model by Kaufman and Beghetto 2009).

Understanding the psychological mechanisms of creative thought on a moment-tomoment basis requires process models. A creative process is a series of mental operations and actions that lead to the generation of ideas or solutions, as well as to novel artifacts, products, or performance (Lubart, 2001). Process models have a long history in the field of creativity. For example, Graham Wallas (1926) proposed that creative processes, consist of four distinct stages that can be separated in time: preparation, incubation, illumination, and verification. Current models of the creative process tend to move away from such distinct stages, and rather emphasize a set of important subprocesses such as 1) problem finding, formulation, and redefinition, 2) divergent thinking, 3) combination and reorganization of information, and 4) analytic-evaluative processes (for a review see Lubart, 2001). One additional benefit of studying creative thought as a process is that it might lead to identification of certain mental operations that could be subject to intervention, such as by improving the efficacy of one stage or the transition from one operation to the next.

Recent work by Oleynick, Thrash and colleagues (2014) highlight an alternative way to segment the creative process: they propose that moments of inspiration are critical pivot points from creative ideation to actualization of an idea. As defined by Thrash and Elliot (2003), creative inspiration is a state of increased motivation for creative activity that has three main characteristics: evocation, meaning that it is often evoked by a stimulus, either an external object or internal thought processes; transcendence, characterized by the feeling of possibilities beyond concrete reality; and approach motivation, a desire to put the evoked 
ideas into the form of a concrete object or action. We note that inspiration shares similarities with insight, the phenomenon of instantaneously "seeing" the solution for a problem rather than solving it analytically: both are related to ideation, and can feel pleasurable. Yet unlike inspiration, insight functions within the context of solving problems with a closed solution (Sternberg \& Davidson, 1995). Moments of inspiration may be elements of the creative process that are amenable to isolation and intervention. Furthermore, as the motivational state and subjective feeling of inspiration arguably result from "illumination" with a highly creative idea (Thrash, Maruskin, Cassidy, Fryer, \& Ryan, 2010), measures of felt inspiration can be taken as an index of the subjective creativity of an idea.

While virtually any object or stimulus could potentially trigger creative inspiration, we propose that intense aesthetic experiences may serve as particularly effective prompts. Many artists and musicians anecdotally cite the creative works of others or the beauty of nature as a source of inspiration for their own creative achievements. For example, art historians note a prominent role of Japanese woodblock prints for the French impressionist movement (e.g. Ives \& Metropolitan Museum of Art, 1974), and a recent study with art students found that both copying and thorough observation of artworks with unfamiliar styles can facilitate creativity in drawing (Okada \& Ishibashi, 2017). Various other studies point at similarities between aesthetic appreciation and creative thought processes. Tinio's "mirror model of art" (2013) proposed that during perception and comprehension of an artwork, an observer engages in similar (though not identical) thought processes as the artist's original creative process, in reversed order. Artistic compositions can trigger strong emotional and physiological reactions (Gerger, Pelowski, \& Leder, 2018; Goldstein, 1980; Schindler et al., 2017; Silvia, 2009; Wassiliwizky, Wagner, Jacobsen, \& Menninghaus, 2015; Zentner, Grandjean, \& Scherer, 2008) similar to those attributed to moments of inspiration (e.g. Doyle, 1998). The occurrence of intense aesthetic experiences can be well characterized by assessing the state of "being aesthetically moved," an exemplary aesthetic emotion (Menninghaus et al., 
2019) sometimes associated with art-elicited chills (Wassiliwizky et al., 2015) that can reflect aesthetic responses derived from differently valenced, or even mixed affective states

(Menninghaus et al., 2015). At the neural level, being aesthetically moved by artwork engages the brain's default-mode network (DMN; Belfi et al., 2019; Vessel, Starr, \& Rubin, 2012, 2013) which has also been associated with trait-level (Beaty et al., 2018; Boccia et al., 2015; Gonen-Yaacovi et al., 2013) and state creativity (Limb \& Braun, 2008).

To test the hypothesis that being aesthetically moved can trigger creative inspiration, we designed an experimental paradigm in which participants wrote short pieces of creative text in response to prompts that varied in their degree of subjective aesthetic appeal.

Following each writing phase, people rated how inspired they felt when beginning their writing (see Figure 1) - our main measure of interest. This task mimics a prompted writing exercise that is used in creative writing workshops, where it is intended to help novice writers learn to generate text with minimal preparation. Our participants were sampled from the general public, and we chose this output modality expecting that a non-artist population would be most comfortable with writing as compared to other possible modes of creative output. Our primary objectives were to 1) operationalize inspiration in an experimental context in a manner that reflected participants' subjective feeling, could be measured on each trial, and could be (at least roughly) isolated in time, and 2) to test whether aesthetically appealing materials are effective primes for creative inspiration. Note that we deliberately focused on a subjective measure of inspiration rather than an objective outcome measure, as we aimed to develop a measure that was more closely linked to a psychological state, unmediated by a participant's ability to translate an inspiring idea into a text that an external judge would rate as creative.

In Experiment 1, we sought to test the ability of an aesthetic prompt (in the most general sense) to trigger creative inspiration compared to a non-aesthetic prompt. Prompts were either highly aesthetically moving paintings (aesthetic prompts), or triads of unrelated 
104 words constructed with no aesthetic intent and expected to have lower aesthetic appeal, but

105 which still contained information that could serve to stimulate writing (non-aesthetic

106 prompts). As aesthetic appeal for artworks is known to be highly idiosyncratic (Vessel,

107 Maurer, Denker, \& Starr, 2018), painting prompts were custom selected for each participant

108 based on their aesthetic ratings from a separate pre-test. We hypothesized that aesthetically

109 moving paintings would lead to higher feelings of inspiration during the writing task than the

110 word prompts.

111 In Experiment 2, prompts consisted only of paintings: paintings that the person

112 previously rated as highly aesthetically moving, paintings they previously rated as non-

113 moving, and novel paintings which were not presented in advance. Here we tested the

114 hypothesis that high-rated paintings would lead to higher feelings of inspiration than low-

115 rated ones, and that there would be no effect of prompt familiarity, resulting in intermediate

116 ratings of inspiration (on average) for the novel paintings. Participants in both experiments

117 also performed an alternative-uses-test (AUT), a common measure of trait-level creativity

118 (Benedek, Könen, \& Neubauer, 2012; Runco \& Acar, 2012) and the amount of produced text

119 was quantified for each trial. Due to logistical reasons, Experiment 1 was conducted in the

120 United States, while Experiment 2 was conducted in Germany; although not intentional, these

121 two populations allow for some degree of generalization across two different cultures. 
prompt

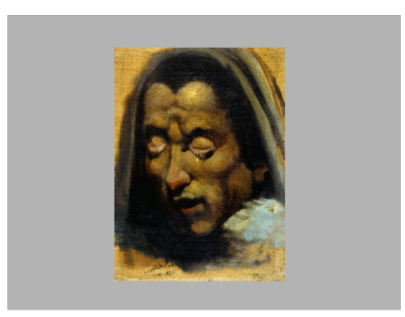

writing phase
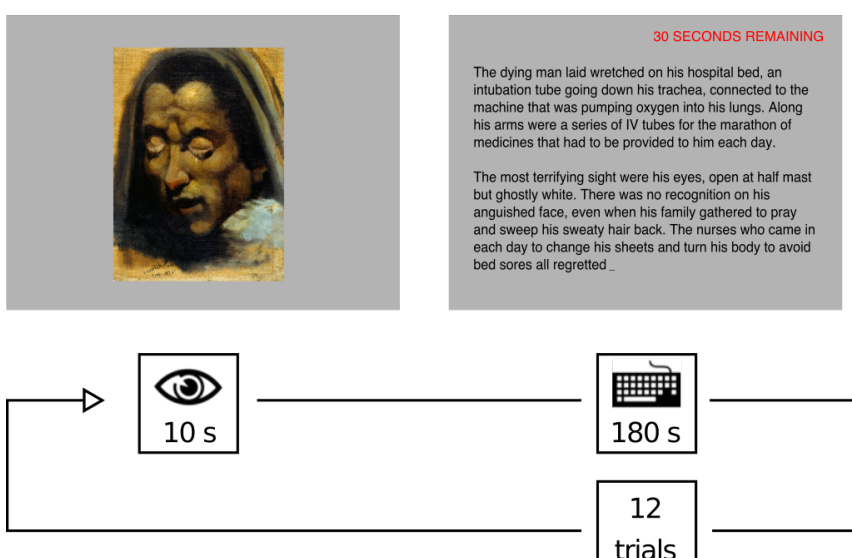

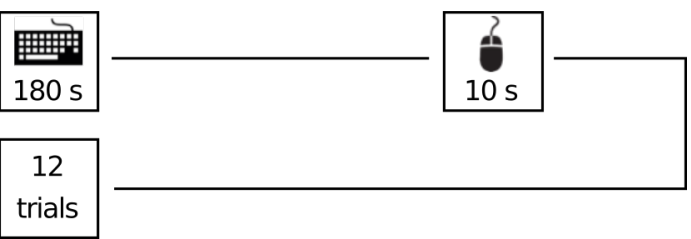

123

Figure 1. Creative writing task for assessing felt inspiration in response to aesthetic prompts. On each trial, a fixation point (not shown) was followed by presentation of a prompt for $10 \mathrm{~s}$. In Exp. 1, these prompts consisted of artworks rated as aesthetically pleasing or of three unrelated-words. In Exp. 2, stimuli consisted of paintings the person previously rated as highly-moving, paintings they previously rated as non-moving, and paintings which were not presented in advance. Participants then had $180 \mathrm{~s}$ to generate an original creative writing sample in response to the prompt. A warning indicated when only 30 seconds remained. Following the writing period, people rated how inspired they felt at the start of the writing period. See Acknowledgments for image credit.

\section{Methods}

\section{Participants}

30 participants took part in the study. Due to an error in the script for artwork selection from the aesthetic pre-rating task that resulted in repeated presentation of the same stimulus in the creative writing task for some participants, four participants had to be excluded from the analysis (reconstruction was not possible), and two have one missing datapoint each but were not removed from the analysis. One other participant was excluded due to non-compliance, leaving data from 25 participants for analysis (15 female, 10 male; age 1855 years, median $=25$ years, mean $=26.6$ years, std $=7.8$ years $)$. Our final sample provided 
143 sensitivity to detect effects of size $d=0.584$ for the main effect of prompt category on

144 inspiration ratings (two-sided, one-sample t-test, with alpha of 0.05 and power of 0.8 ).

145 We applied convenience sampling: participants were recruited using flyers posted on

146 the campuses of New York University and received monetary compensation. Participation

147 was limited to persons fluent in written English. The study was run in a group testing room in

148 groups of two to four people. The experimenter was present in the room during the

149 experiment. Besides the participants and the experimenter, no one was present during data

150 collection. The experimenter supervising the data collection was aware of the experimental

151 conditions and study hypothesis during data collection. Each participant provided informed

152 written consent before the beginning of the experiment. The study was approved by the New

153 York University Committee on Human Subjects and adhered to the ethical standards of the

154 Declaration of Helsinki. Data were collected from Apr. 2015 to Dec. 2015.

\section{Stimuli}

157 Paintings - the stimuli consisted of twenty paintings selected from a larger stimulus

158 set of 109 paintings collected for an earlier study (Vessel et al., 2012). The set contained

159 selected works from the Catalog of Art Museum Images Online (CAMIO; note that this

160 database was closed Dec. 2018 and cannot be accessed anymore) and included paintings from

161 a variety of cultural traditions and historical periods; images that are commonly reproduced

162 were not included in order to minimize the possibility of recognition. We subselected

163 paintings for the present study based on data from a previous aesthetic rating study of the

164 larger stimulus set $(\mathrm{N}=20$ raters; data published in Vessel et al., 2018) for having an average

165 aesthetic rating ("how strongly does this painting move you," see below) of at least 4 on a 7-

166 point Likert scale and were rated 5.5 or higher by at least $30 \%$ of the 20 raters; see 
Table A2 for a list of the selected paintings. Images were scaled not to exceed $20^{\circ}$ of visual angle, and the area on screen did not exceed $75 \%$ of a $20^{\circ}$ box.

Word Triads - Six triads of unrelated word were selected from a stimulus set used in a Remote Associates Test (Bowers, Regehr, Balthazard, \& Parker, 1990). We took the six word triads reported as being the least coherent in the original study; An incoherent word triad is defined as a set of three words that has no common remote associate (Bolte, Goschke, \& Kuhl, 2003); e.g. "trumpet, bathtub, grant". Word triads have been successfully used as prompts for creative writing in past experiments (Howard-Jones, Blakemore, Samuel, Summers, \& Claxton, 2005). The full set used in the present study is enclosed in Table Al. Participants were not exposed to the word triads prior to the creative writing task.

\section{Procedure}

Exp. 1 was conducted in English at New York University in New York, NY. At the start of the experiment, each participant completed a set of questionnaires: a demographic background questionnaire, an abbreviated version of the Positive and Negative Affect Schedule (PANAS-X; Watson \& Clark, 1999), the State-Trait Anxiety Inventory (STAI-Y; Grös et al., 2007; Julian, 2011; Spielberger, 1983), and the Aesthetic Responsiveness and Engagement Assessment (AReA; Schlotz et al., 2020), in their English version using pen and paper. The AReA questionnaire is a tool developed by our group to measure participants' general aesthetic responsiveness with items assessing the frequency with which people experience three relevant states: aesthetic appreciation, intense aesthetic experiences, and creative engagement. The remainder of the experimental session consisted of four phases: 1) an alternative-uses task (AUT) to measure trait creativity, 2) an aesthetic rating pre-test, 3 ) the main creative writing task to measure felt inspiration (see Figure 1), and 4) an aesthetic rating post-test. Both aesthetic rating tasks required the participants to rate all twenty artwork stimuli. All tasks (except questionnaires) were implemented and run using MATLAB 2013b 
193 (The MathWorks) and the PsychToolbox v3 (Brainard, 1997) on Microsoft-Windows PC's

194 (Microsoft Corporation). Instructions, text-stimuli and images of paintings were presented on 195 calibrated Dell P190S computer monitors. Participants were placed at a distance of 16”

196 (approx. $41 \mathrm{~cm}$ ) from the monitors. Before each task, people were shown a series of

197 instruction screens explaining the task and the measure used. The experimenter read the full

198 set of instructions to the group as a whole, and participants could ask questions before the 199 beginning of each task (see Online Supplementary for full instructions). A full debriefing was 200 performed at the end of the experiment. Overall, the session took about $90-120$ minutes 201 depending on each participant's individual speed. AUT Ideational Fluency - The Alternative-Uses Task (AUT; Guilford et al., 1978) is a 203 divergent thinking task that is frequently used as a measure for trait-level creativity (Cousijn, 204 Zanolie, Munsters, Kleibeuker, \& Crone, 2014; Friedman \& Forster, 2001). We implemented 205 the AUT "brick" paradigm and scored for ideational fluency, a reliable and robust method for 206 scoring the AUT (Martindale, Hines, Mitchell, \& Covello, 1984). Participants had three 207 minutes to come up with as many unusual uses as possible for the prompt "a single brick", 208 which were typed on a computer keyboard into a simple text editing interface. Responses were evaluated by the experimenter - ordinary, impossible, or nonsense replies were not counted. Fluency was scored as the total number of valid responses. Aesthetic Rating Tasks (Pre-/Post) - Participants rated a set of 20 paintings on the 212 question "How strongly does this painting move you?" using a continuous response scale. 213 Prompts were presented in pseudo-randomized order to counterbalance image order effects.

214 People were instructed by the experimenter to make ratings based on their feelings at the 215 moment, without regard to ratings made on earlier trials. Being aesthetically moved is a 216 description of aesthetic experience that is often used by researchers in the field of empirical 217 aesthetics (Armstrong \& Detweiler-Bedell, 2008; Menninghaus et al., 2015; Silvia, 2009; 218 Vessel et al., 2012). It is a term that accounts for the possibility that artworks which are not 
stereotypically beautiful can also evoke powerful and emotionally moving responses.

Participants were given two practice trials to gain familiarity with the types of stimuli used and the rating scale. These practice stimuli were not used in the main experiment. After having finished the following writing task (see below) they repeated the aesthetic rating task of the same 20 stimuli in a different order. Each trial began with a blank screen $(1 \mathrm{~s})$ followed by a fixation point $(0.5 \mathrm{~s})$, an image of an artwork $(6 \mathrm{~s})$, a blank screen, and a rating scale. Participants were asked to indicate their ratings on a continuous scale by positioning a visual slider moving the mouse up and down; responses were locked in by clicking the mouse button. Participants were instructed "If the image was highly moving, then use the mouse to position the slider cursor towards the high end of the scale (marked " $\mathrm{H}$ ") and click the mouse to lock in your response. If the painting did not move you at all, move the slider to the low end of the scale (marked "L") and lock in your response. If you did not feel strongly one way or the other, use the middle of the scale" (see Online Supplementary for full instructions).

232 Responses were saved as numerical values ranging from 0 (low) to 1 (high). The continuous 233 rating scale had a response precision of 0.001 , technically limited by the number of pixels on 234 the scale. Movement direction of the mouse corresponding to the respective ends of the scale was randomized between participants. Participants were instructed to use the entire scale.

236 Image order was randomized across participants, with pairs of participants receiving mirror 237 reversed orders of each other to counteract any order effects. Different random orders were 238 used for the pre- and post- aesthetic rating tasks. Creative Writing Task - Participants were asked to produce short pieces of creative

240 writing in response to 12 prompts (see Figure 1). The response variable of interest was the 241 self-reported felt inspiration in each writing period. The prompts belonged to two conditions: 242 "aesthetic" prompts consisting of the six paintings rated highest by the participant in the 243 preceding aesthetic rating task, and "non-aesthetic" prompts consisting of six unrelated word triads. Prompts were presented in pseudo-randomized order with condition alternating by trial 
245 (half of the participants starting with art prompts, half of them starting with text prompts). On 246 each trial, a fixation cross was presented for $0.5 \mathrm{~s}$, followed by the prompt for $10 \mathrm{~s}$. After 247 stimulus presentation, people had $180 \mathrm{~s}$ to produce text in response to the prompt using a 248 basic text editor interface that allowed for typing, deletion and addition of line breaks.

249 Participants were asked to not simply describe the prompt or their reaction to it but to produce 250 a series of "independent, short creative writing samples." (For full instructions see Online 251 Supplementary. At the beginning of the session there was a practice trial with a separate 252 stimulus to practice using the text-editor.) After the end of each writing period, participants 253 were asked to answer the question "How strongly were you inspired?" on a continuous scale 254 with the ends marked "H" or "L" (instruction: "Use the far right of the scale if you felt highly 255 inspired, the far left if you were not inspired at all, and the middle if you didn't feel strongly one way or the other.”). Participants were asked to judge the feeling of inspiration they had while starting their creative process, and were told not to judge the prompt itself, nor to judge their final product (see Online Supplementary for full instructions). A similar self-report measure was used in Thrash and Eliot's Inspiration Scale, who let their participants assess the statement "I feel inspired" in terms of Frequency and Intensity, using a 7-point Likert scale.

261 They found the scale to be internally consistent, with an average Cronbach's $\alpha$ of 0.92 262 (Thrash \& Elliot, 2003). After a rating was provided, there was a $30 \mathrm{~s}$ break before the next 263 prompt was shown. After six trials participants were offered a longer, self-paced break.

\section{Data analysis}

Data analysis and visualization was done using MATLAB 2018a (The MathWorks) and R (R Core Team, 2018) on a Macintosh PC (Apple Inc.). For visualization of mean 267 ratings we used a customized version of the boxPlot function from the IoSR MatlabToolbox 268 (https://github.com/IoSR-Surrey/MatlabToolbox). Descriptive statistics were computed using

$269 \mathrm{R}$ with built-in functionality, t-tests were generally two-sided, computed with R's t.test 270 function, effect sizes were computed using cohen.d function from R's effsize package. The 
main hypotheses (effect of prompt category) were tested using linear mixed effects regressions with the lmer function from lme4 package in $\mathrm{R}$ (Bates, Mächler, Bolker, \& Walker, 2015). Participants' rated inspiration was modelled as the dependent variable, with a fixed effect of prompt category and two random effects of participant and stimulus. To assess the overall significance of all reported linear models, Likelihood Ratio Tests were performed: LMM-coefficients were compared to reduced intercept-only models (no fixed effect, random effects of participant and stimuli) using R's anova function. Power analysis was performed using G*Power v3.1 (Faul, Erdfelder, Lang, \& Buchner, 2007).

\section{Results and Discussion}

Previous research has shown that different people have highly idiosyncratic aesthetic tastes for visual artworks (Vessel et al., 2018, 2012), making it impossible to identify a single set of artworks that all observers would find aesthetically moving. We therefore included an aesthetic rating pre-test before the creative writing task. Our participants viewed 20 paintings and rated them for how strongly the paintings moved them aesthetically (see Methods and Online Supplementary). The six top-rated paintings for each observer were used as prompts in the subsequent writing task. All 20 stimuli were among the top six for at least two participants. On average, each painting was selected by $7.4(\mathrm{SD}=2.66)$ of the 25 observers and the overall count of the stimuli followed a normal distribution (a Shapiro-Wilk normality test on frequency counts of the paintings did not reject the null hypothesis: $W=0.94791, p=$ 0.3365). The paintings selected for the writing task had an average aesthetic rating of $\mathrm{M}=$ $0.79,95 \% \mathrm{CI}[.774, .813]$ across participants (0 to 1 response interval). The word triads were not rated by the participants.

Consistent with our hypothesis, inspiration ratings in the writing task were significantly higher for trials with aesthetically moving paintings (aesthetic prompts) than for trials with word triads (non-aesthetic prompts; Figure 2). The grand mean inspiration rating in aesthetic trials $(\mathrm{M}=0.63,95 \% \mathrm{CI}[.591, .675])$ was higher than the grand mean rating in non- 
aesthetic trials $(\mathrm{M}=0.48,95 \% \mathrm{CI}[.438, .532])$. All individual ratings of inspiration were used to fit a linear mixed effect regression model with fixed effect of stimulus category and $p<0.002)$.

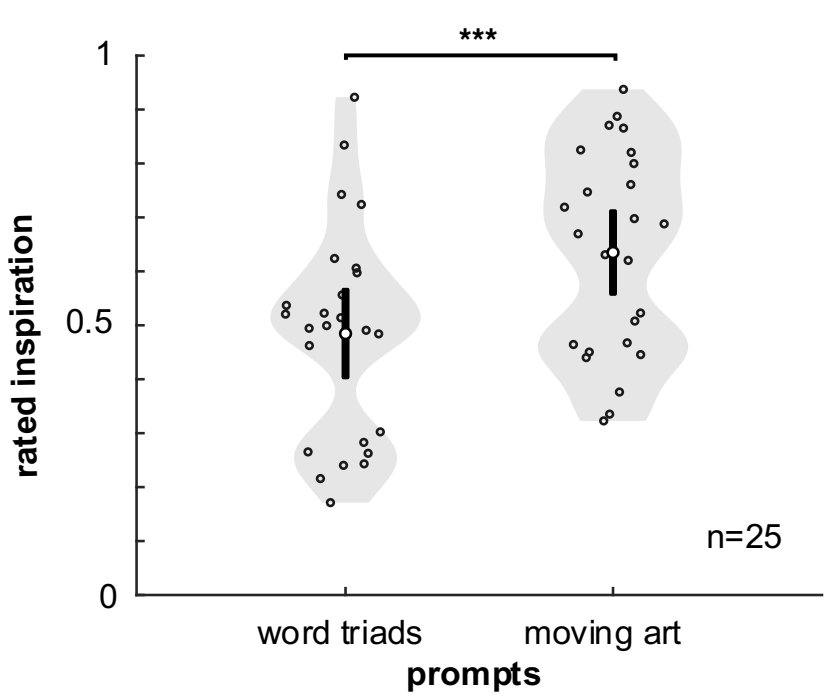

Figure 2. Higher felt inspiration ratings in Exp. 1 when participants wrote in response to aesthetically moving prompts. Each dot represents the mean inspiration rating of one participant for writing trials with either unrelated word triads (non-aesthetic) or paintings rated as aesthetically moving (aesthetic). $\mathrm{n}=25$. White dot and black bar indicate grand mean with $95 \%$ confidence interval. $* * \mathrm{p}<0.001$ (significance level from linear model). Gray shaded area represents the data density computed using a gaussian kernel with a width that is optimal for normal data.

313 second fixed effect with interactions to a new model otherwise identical to the LMM

314 described above. AUT score had no significant effect on inspiration rating (slope $=0.013$, SE

$315=0.013, \mathrm{p}=0.311$ using Satterthwaite's degrees-of-freedom method), but there was a significant interaction between AUT score and category (slope $=-.023, \mathrm{SE}=0.009, \mathrm{p}<0.02$ 
317 using Satterthwaite's degrees-of-freedom method): people with lower AUT scores tended to

318 show a larger effect of category on inspiration ratings. A Likelihood Ratio Test against the 319 previously described model without fixed effect of AUT was significant $\left(\chi^{2}(2)=6.27, p<\right.$ $320 \quad 0.05)$.

\section{Experiment 2}

The "aesthetic" and "non-aesthetic" prompts of Exp. 1 differed in several ways. Most prompts consisted of written stimuli - the two conditions thus differed on a variety of other dimensions besides their aesthetic properties that may also have accounted for the observed behavioral differences. In addition, the artworks were rated during the aesthetic pre-test and were thus familiar to the participants, whereas the word triads were novel. To address these potential confounding factors, we conducted a second experiment in which the potential effect of stimulus modality (visual art vs. text) was eliminated by only using visual art, and the effects of aesthetic appeal and familiarity were directly examined. Exp. 2 thus contained three prompt categories: aesthetically moving visual artworks, non-moving visual artworks (both determined via an aesthetic rating task that preceded the writing task), and a set of novel artworks that had not been previously shown to the participants.

\section{Methods}

\section{Participants}

41 participants took part in the study. Sample size was powered for detection of a

339 moderate effect size $(\mathrm{d}=0.5)$ for the main effect of prompt category on inspiration ratings

340 using a two-sided, one-sample t-test, alpha of 0.05 and power of 0.8 . Two participants did not

341 finish data collection due to software failures and were excluded from analysis, one was 
342 excluded because their distribution of ratings in the aesthetic pre-test did not allow for the

343 identification of 4 non-moving stimuli, and four because they gave zero valid responses in the

344 AUT task, leaving 34 participants for analysis (21 female, 13 male; age 19-53 years, median

$345=24.5$ years, mean $=27.3$ years, $\mathrm{std}=8.7$ years $)$. Our final sample provided sensitivity to

346 detect effects of size $d=0.495$ or larger (two-sided, one-sample t-test, with alpha of 0.05 and 347 power of 0.8$)$.

348 We applied convenience sampling: participants were recruited via flyers posted on the 349 campuses of Goethe University (Frankfurt, Germany) and Johannes-Gutenberg University 350 (Mainz, Germany), as well as direct mailings to subscribers of an institute-hosted participant 351 database (>1500 members, open to everybody to subscribe) and received monetary

352 compensation. Participation was limited to persons fluent in written German, age 18-55 years, 353 with normal or corrected vision. Participants performed the experiment individually in an 354 acoustically and visually shielded cabin. The experimenter was present outside the cabin and 355 could be contacted via intercom. Besides the participants and the experimenter, no one was 356 present during data collection. Two different experimenters collected the data. The 357 experimenters supervising the data collection were aware of the experimental conditions and 358 study hypothesis during data collection. Each participant provided informed written consent 359 before the beginning of the experiment. The study was approved by the Ethics Committee of 360 the Max Planck Society and adhered to the ethical standards of the Declaration of Helsinki.

361 Data were collected from Nov. 2017 to Feb. 2018.

\section{Stimuli}

364 Twenty-four paintings were selected as stimuli: Twenty of the paintings were identical

365 to those used in Exp. 1 and were presented in the aesthetic rating pre test. An additional 4 366 paintings were selected from the larger set of 109 painting to be used as "novel" (i.e. not

367 previously presented) paintings only in the creative writing task (and the aesthetic rating post- 
368 test). As for the 20 other paintings (described above), selection was based on the rating

369 dataset from Vessel et al. (2018), choosing the 4 paintings with the highest variance across

370 participants among the twenty paintings with aesthetic ratings closest to the grand mean rating

371 (3.6 on a 7-point scale). A full list of the paintings used in this experiment is available in 
Table A2.

\section{Procedure}

The overall procedure was almost identical to that of Exp. 1; however, Exp. 2 was conducted in German at the Max Planck Institute for Empirical Aesthetics in Frankfurt, Germany and all task instructions required translation to German. Participants read through the instructions at their own pace. In addition to the questionnaires from Exp. 1 (PANAS, STAI, AReA), each participant completed the Snaith-Hamilton-Pleasure-Scale (SHAPS;

380 Snaith et al., 1995). Questionnaires were presented in their German version. All tasks (except questionnaires) were run using MATLAB 2017a (The MathWorks) and the PsychToolbox v3 (Brainard, 1997) on Microsoft-Windows PC's (Microsoft Corporation). Instructions, textstimuli, and images of paintings were presented on a Benq XL2420Z computer monitor. Participants were placed at a distance of $60 \mathrm{~cm}$ from the monitors. Overall, the session took about $90-120$ minutes depending on the speed of the participants.

AUT Ideational Fluency - The task and analysis was identical to that described in Exp.

1, using the German version of the "brick" paradigm (prompt: "ein einzelner Ziegelstein“). Aesthetic Rating Tasks (Pre-/Post) - For the pre-rating task, participants rated the aesthetic appeal of 20 artworks using the exact same task described in Exp. 1. Since

390 participants were German the question "Wie ansprechend finden Sie dieses Gemaelde?"

391 ("How appealing was this painting to you") was displayed. This translation was chosen over

392 the literal German translation of "moving" - "bewegend" - since this was flagged as

393 potentially problematic for application with a continuous scale. The ends of the rating scale

394 were marked with "+" (high) and "_“ (low). In contrast to Exp. 1, the aesthetic rating post-test

395 (after the writing task) required participants to rate the twelve pictures used as writing 396 prompts (see below). For half of the participants presentation order was the same as in the

397 writing-task, for the other half it was reversed. 
Creative Writing Task - The procedure was identical to Exp. 1, but prompts consisted exclusively of paintings. The twelve writing prompts were from three conditions: the four paintings rated highest by the participant in the aesthetic rating pre-test ("moving"), the four paintings rated lowest in the pre-test ("non-moving"), and four paintings that were not rated in advance ("novel", the same for all participants). Prompt-order followed a predefined scheme which balanced transitions between the different categories. This scheme was reversed for half of the participants. After writing, participants answered the question "Wie inspiriert haben Sie sich beim Schreiben gefuehlt?“ ("How inspired did you feel during writing") using 406 the same slider used in the aesthetic pre-rating (ends marked "+" / "-" for "high" and "low" 407 inspiration) with the instruction: "If you have felt very inspired position the slider cursor at 408 the high end of the scale (marked "+"). If you have felt uninspired, position the slider at the 409 low end of the scale (marked "-"). If you do not have a strong tendency one way or the other, 410 use the middle of the scale" (see Online Supplementary for full German instructions).

411 Participants were presented with two practice-prompts (novel paintings) to practice using the 412 editor and rating scale (30 seconds writing each).

\section{Data analysis}

414 Data analysis was largely analogous to Exp. 1. Due to the slightly different 415 experimental design, the main linear model was adapted: participants' rated inspiration was 416 still modelled as the dependent variable, with a fixed effect of prompt category and two 417 random effects of participant and stimulus. Yet, to directly test effects of aesthetic appeal and 418 pre-exposure on felt inspiration, linear contrasts were computed for the conditions novel vs. 419 familiar (collapsing across moving and non-moving prompts) and for moving vs. non-moving 420 (ignoring novel prompts) using R's MASS package. Specific significance values for the two 421 linear contrasts were estimated using Satterthwaite's degrees-of-freedom method implemented 422 in R's lmerTest package (Kuznetsova, Brockhoff, \& Christensen, 2017). A second model of 
inspiration ratings for only the novel stimuli was fitted with fixed effect of post-test aesthetic ratings and random effects of participant and stimulus.

\section{Results}

Of the 20 stimuli presented in the aesthetic rating pre-test, only one was not among the top four (aesthetically moving) stimuli for any participant. All others were selected by at least two participants. On average, each painting was selected by $6.8(\mathrm{SD}=4.37)$ of the 34 observers. A Shapiro-Wilk normality test on frequency counts of all aesthetically moving paintings did not reject the null hypothesis $(W=0.93452, p=0.1885)$, indicating that the overall count for moving stimuli followed a normal distribution. All 20 were among the bottom four (non-moving) stimuli for at least one participant. On average, each painting was selected by $6.8(\mathrm{SD}=5.96)$ of the 34 observers. Yet for non-moving paintings the ShapiroWilk normality test on frequency counts of the paintings did reject the null hypothesis $(W=$ $0.8038, p<0.001)$. The distribution appears skewed to the left - most paintings (12 out of 20) were selected by only 5 or fewer participants. The moving paintings had an average aesthetic rating of $\mathrm{M}=0.86,95 \% \mathrm{CI}[.838, .876]$, while the non-moving paintings had an average aesthetic rating of $\mathrm{M}=0.20,95 \% \mathrm{CI}[.180, .226]$. For comparison, the novel paintings, assessed only after the writing task in a post-test rating task, had an average aesthetic rating of $\mathrm{M}=0.50,95 \% \mathrm{CI}[.455, .551]$. A two-sample independent $\mathrm{t}$-test (two-sided) comparing aesthetic ratings of moving and non-moving conditions was significant, $t(260.4)=43.24, p<2$ x $10^{-16}, 95 \%$ CI $[.624, .683]$, Cohen's $d=5.24,95 \%$ CI $[4.740,5.746]$.

\section{Participants reported higher felt inspiration during writing trials prompted by} aesthetically moving artworks than by trials prompted by non-moving artworks (Figure 3a), while ratings following novel paintings did not significantly differ from the ratings following pre-exposed paintings (i.e. both moving and non-moving conditions). The mean inspiration rating for moving paintings $(\mathrm{M}=0.64,95 \% \mathrm{CI}[.596, .683])$ was higher than the mean rating for non-moving paintings $(\mathrm{M}=0.49,95 \% \mathrm{CI}[.441, .530])$, while mean inspiration ratings for 
novel paintings $(\mathrm{M}=0.58,95 \% \mathrm{CI}[.533, .624])$ lay in between. Individual ratings of

inspiration were fit using a linear mixed effect regression model with fixed effect of stimulus

453 Satterthwaite's degrees-of-freedom method) but the contrast of familiar (moving and non-

454 moving) vs. novel was not (slope $=0.018, \mathrm{SE}=0.045, p=0.705)$. A Likelihood Ratio Test of 455 the full model against an intercept-only model with the same random effects was significant

a)

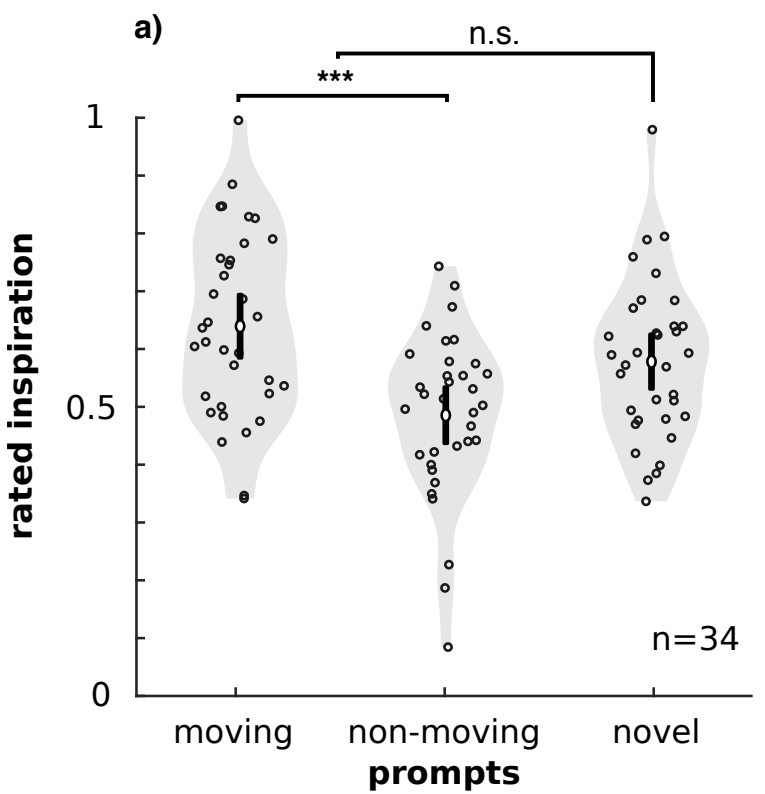

b)

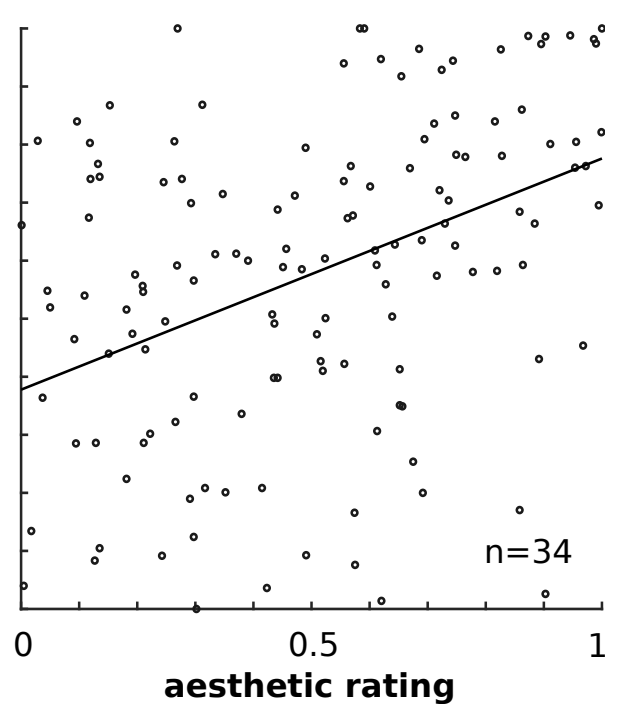

Figure 3. Aesthetic appeal but not stimulus familiarity influenced felt inspiration in Exp. 2. a) Mean

inspiration ratings were higher when participants wrote about aesthetically moving paintings than when they wrote about non-moving paintings. Writing trials with novel paintings as prompts produced intermediate ratings of inspiration; a linear contrast of familiar (moving and non-moving) vs. novel was not significant. Each open circle represents the mean inspiration rating per category of one participant $(n=34)$. White dot and black bar indicate grand mean and $95 \%$ confidence interval. ${ }^{* *} \mathrm{p}<0.001 ;$ n.s. indicates $\mathrm{p}>0.05$ (significance level from linear model). Gray shaded area represents the data density computed using a gaussian kernel with a width that is optimal for normal data. b) Even for novel paintings, there was a positive linear relation between rated inspiration and rated aesthetic appeal in the aesthetic post-test. Each dot represents a single trial (four per 
468

469

470

471

472

473

474

475

476

477

478

479

480

481

482

483

484

485

486

487

488

489 participant, 136 observations total). Regression line computed using linear modelling with fixed effect of posttest aesthetic rating and random effects of participant and stimulus (slope $=0.398$, intercept $=0.378)$.

While average inspiration ratings for novel stimuli did not significantly differ from the average ratings following the pre-exposed (familiar) artworks, we did observe a linear relationship between inspiration ratings following novel stimuli and subsequent (post-test) ratings of aesthetic appeal (Figure 3b). We fit a linear mixed effect regression model with fixed effect of post-test aesthetic ratings and random effects of participant and stimulus on inspiration ratings for all novel stimuli (see Methods) and found a significant positive relationship between aesthetic ratings and rated inspiration (slope $=0.398, \mathrm{SE}=0.074$, intercept $=0.378, \mathrm{SE}=0.052$, Likelihood Ratio Test against an intercept-only model with the same random effects, $\left.\chi^{2}(1)=23.8 \mathrm{p}<0.000002\right)$.

To investigate the role of trait-level creativity, AUT-fluency scores were added as a second fixed effect with interactions to a new model otherwise identical to the above described LMM. The model yielded a negative effect of AUT score on inspiration rating (Slope $=-0.011, \mathrm{SE}=0.005, \mathrm{p}<0.04$ using Satterthwaite's degrees-of-freedom method), and none of the interactions with the category contrasts reached significance (interaction with aesthetic appeal: Slope $=-.011, \mathrm{SE}=0.009, \mathrm{p}=0.231 \mathrm{using}$ Satterthwaite's degrees-offreedom method; interaction with preexposure: Slope $=-.0002, \mathrm{SE}=0.008, \mathrm{p}=0.983$ using Satterthwaite's degrees-of-freedom method). A Likelihood Ratio Test against the previously described model without AUT was not significant $\left(\chi^{2}(3)=5.73, p=0.125\right)$. 


\section{Correlation of participant-level and single-trial-level measures in}

\section{pooled data}

We performed exploratory analyses to investigate the connections between inspiration, aesthetic appeal and text length on a trial-by-trial basis, and to assess potential links between participant states and traits (like mood, or hedonic and aesthetic responsiveness) and the observed behavior. We characterized the relationships between inspiration, aesthetic appeal and a number of additional measures by computing a series of pairwise correlations.

Correlated measures were either attributes of a participant (participant level: age, AUT score, questionnaire scores, average number of typed characters) or corresponding responses to a stimulus by one participant (single-trial level: aesthetic ratings from pre- and post-sessions, inspiration rating, number of typed characters). To increase the statistical sensitivity for this analysis we decided to pool data from both experiments together, but only those trials collected under largely identical conditions - i.e. trials with painting stimuli that were rated for aesthetic appeal before and after the writing session (thereby excluding word prompts from Exp. 1 and novel paintings from Exp. 2.)

\section{Data pooling}

We statistically compared the population characteristics and the distributions of ratings for the set of paintings designated as aesthetically moving in the two studies (identical tasks) and decided that observed differences should not preclude a pooling of the data (see Appendix B - Across study comparison for additional information). The partial pooling (“moving artworks" from Exp. 1, "moving" and "non-moving artworks” from Exp. 2) resulted in a total of 420 trials from $n=59$ participants ( 36 female, 23 male; age 18-55 years, median $=25$ years, mean $=27.0$ years, std $=8.3$ years $)$. The pooled sample provided sensitivity to detect effect sizes of $r= \pm 0.355$ or bigger on the subject level, and $r= \pm 0.136$ or 
515 bigger on the single-trial level (two-sided, Pearson correlation, with alpha of 0.05 and power

516 of 0.8 ). Note that correction for multiple testing raised the individual significance thresholds

517 above this value.

518 Data analysis

Pearson's R for all possible combinations of participant-level factors were computed using R's rcorr function from Hmisc package (Harrell, n.d.). Participant-level factors included the participant's age, AUT-fluency score, scores on mood and personality trait measures (PANAS, STAI, AReA, SHAPS; see Exp. 1 Methods), and each participant's average text length, computed as the total number of typed characters of each short response (averaged over all 12 trials). Correlation coefficients for all possible combinations of singletrial-level variables were computed using the repeated measures correlation algorithm implemented in R's rmcorr package (Bakdash \& Marusich, 2017) to take into account that individual observations were clustered by participant. Single-trial-level measures included aesthetic pre-rating, inspiration rating, text count (total number of typed characters in the trial), and aesthetic post-rating. was implemented using R's corrplot function from the corrplot package (Wei \& Simko, 2017). In the General Discussion, correlation coefficients were statistically compared using Hotelling's (dependent groups) and Fischer's (independent groups) method implemented in R's cocor package (Diedenhofen, 2016).

Additionally, we performed a mediation analysis to test whether a potential effect of aesthetic appeal on amount of text produced was a direct effect, or rather an indirect effect 538 mediated by rated inspiration. A full mediation model was constructed from a simplified 539 model for the dependent variable (main effects of rated inspiration and aesthetic rating on amount of text produced) and mediator model (main effect of aesthetic rating on rated 
541 inspiration) using R's mediation package (Tingley, Yamamoto, Hirose, Keele, \& Imai, 2014).

542 Significance of the indirect effect was tested using bootstrapping procedures. Unstandardized

543 indirect effects were computed for each of 10,000 bootstrapped samples, and the $95 \%$

544 confidence interval was computed by determining the indirect effects at the 2.5 th and 97.5 th

545 percentiles.

546

547 Results and discussion

Results of the pairwise correlation of participant-level factors are shown in Figure 4a:

549 Only two correlations were found to be significant at the $p<0.05$ level (Holm corrected):

550 State and trait anxiety measures were highly positively correlated $(r=0.63)$; Positive affect

551 (PA) was negatively correlated with state anxiety measures $(r=-.47)$. The full set of

552 correlation coefficients is reported in Table $\mathrm{C} 1$.

553 Results of the pairwise repeated measures correlation of all single-trial-level responses

554 are shown in Figure 4b: All measures were positively correlated with each other, yet to

555 different degrees and not always significantly (Holm corrected $p<0.05$ ). The highest

556 correlation was observed between pre- and post-test aesthetic ratings ( $r=0.78)$. Inspiration

557 ratings were significantly correlated with pre- and post-test aesthetic ratings, though to a

558 higher degree with the post rating (pre: $r=0.31$, post: $r=0.45$ ). Text count was positively

559 correlated with the other measures, but only the relationship to inspiration rating reached

560 significance $(r=0.42)$. The full set of correlation coefficients is reported in 
Table C2.

A mediation analysis indicates that the relationship between aesthetic rating (pre) and amount of text produced was fully mediated via rated inspiration. Results are shown in Figure

5. The standardized regression coefficient between aesthetic rating and inspiration rating was statistically significant (standardized beta $=0.319, \mathrm{p}<0.001$ ), as was the standardized regression coefficient between inspiration rating and amount of text produced (standardized beta $=0.213, p<0.001)$. According to bootstrapping results the standardized indirect effect was statistically significant (standardized beta $=0.068, \mathrm{p}<0.001$ ). The standardized total

569 effect of being aesthetically moved on amount of text produced was not significant

570 (standardized beta $=0.082, \mathrm{p}=0.093$ ) and the remaining direct effect was 0.014 .

\section{a) Participant level}

$n=59$

(n)

\section{AUT \\ AReA}

PA

NA

STAls

STAIt

SHAPS

mTXT

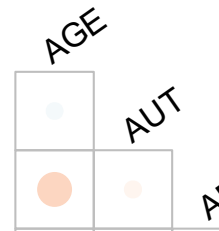

\section{b) Single-trial level}

$$
n=420
$$

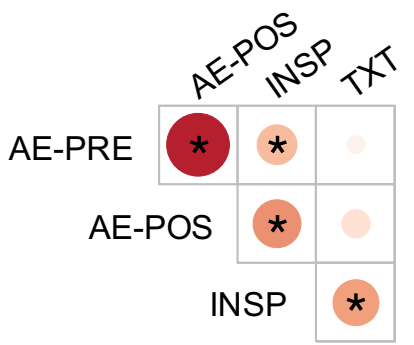

pl
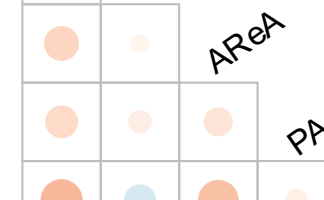

NP

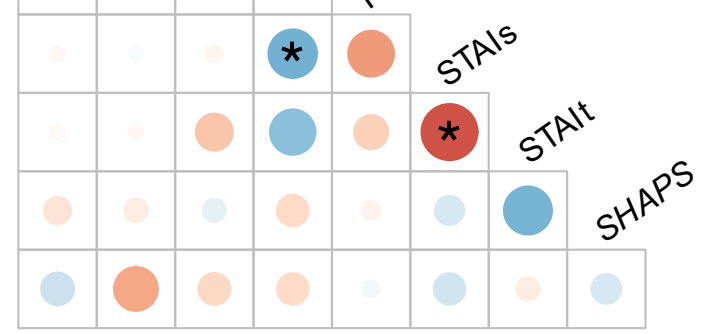

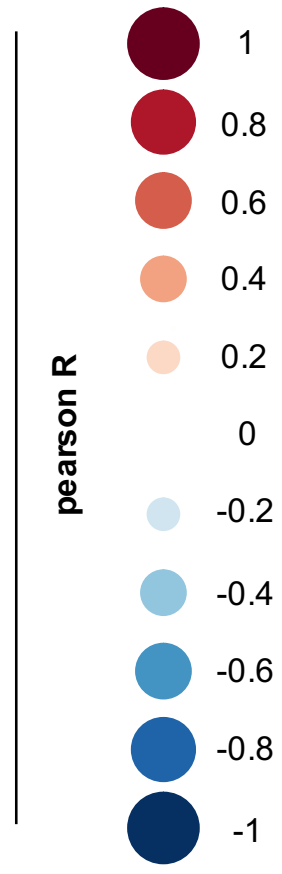

Figure 4. Associations between (a) participant-level factors and (b) single-trial-level response parameters in pooled data (painting prompts only). a) Pairwise correlations of participant-level data for all participants (n

$575=59$ ) from Exp. 1 and 2, including AGE, AUT-score, questionnaire scores (AReA; PA, NA - PANAS 
coefficients (Pearson R) are color and size coded; red - positive, blue - negative, larger and more saturated circles indicate higher values. Significant correlations $(\mathrm{p}<0.05$, Holm-corrected) marked with *. Note that SHAPS data was only collected in Exp. 2. b) Pairwise repeated-measures correlations of single-trial-level measures $(n=420$ trials from 59 participants): inspiration ratings (INSP), text count (TXT), and corresponding aesthetic ratings from pre- and post-tests (AE-PRE/AE-POS). Only data from trials with all four measures were included: moving paintings from Exp. 1 and moving and non-moving (familiar) paintings from Exp. 2.

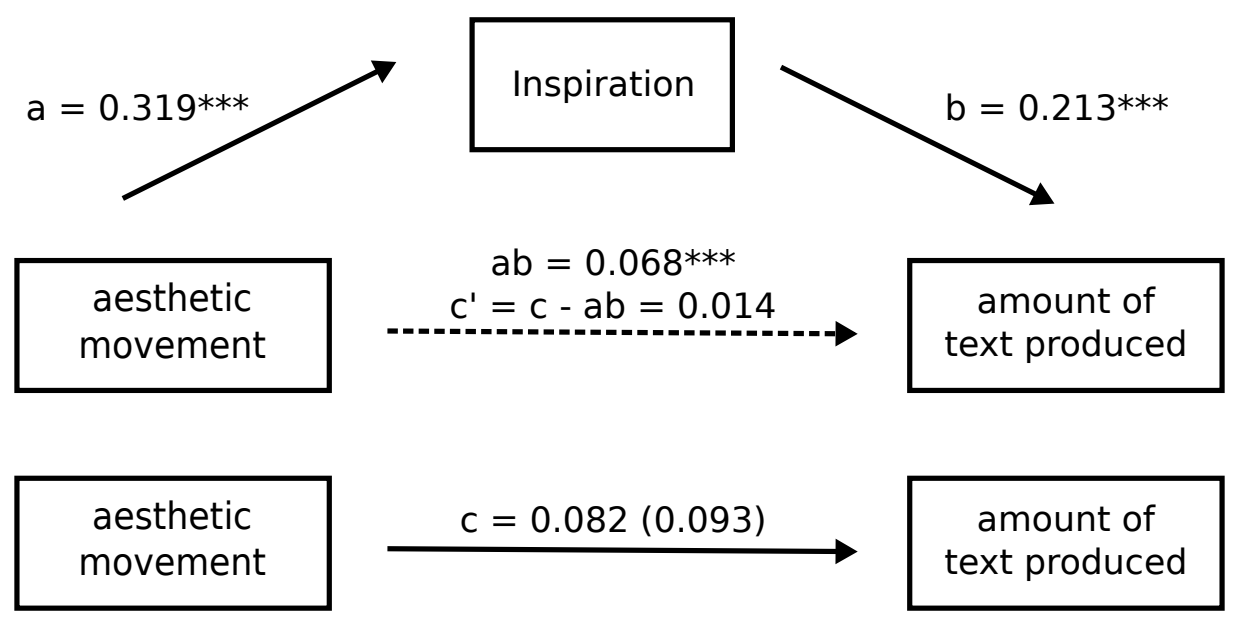

Figure 5. mediation analysis. The mediation model suggests that the relationship between aesthetic rating and amount of text produced was fully mediated by rated inspiration. a - standardized effect of aesthetic rating on the mediator, $\mathrm{b}$ - standardized effect of the mediator on amount of text produced, $\mathrm{c}$ - standardized total effect of aesthetic rating on amount of text produced, $a b$ - standardized indirect effect of aesthetic rating on amount of text produced, c' - standardized direct effect of aesthetic rating on amount of text produced (total effect minus indirect effect). $* * * \mathrm{p}<0.001$

\section{General Discussion}

Moments of inspiration represent elements of the creative process that may be particularly amenable to intervention. Using a creative writing paradigm, we found that aesthetically appealing artworks induced higher felt inspiration than non-aesthetic word triads and non-appealing artworks. In Exp. 1, writing about aesthetically appealing artworks resulted in higher ratings of felt inspiration than writing about prompts consisting of three non-related words, which still contained information that could serve to stimulate writing, but had no 
artistic intent. Exp. 2 found that high versus low aesthetic appeal, and not preexposure to the specific prompts, was the factor associated with higher ratings of felt inspiration. Writing about aesthetically appealing (moving) artworks resulted in higher inspiration ratings than writing about non-moving artworks, while novel artwork prompts led to intermediate ratings of felt inspiration that were not different from the average of the familiar (moving plus nonmoving) artworks. This experiment also ruled out potential confounds due to the different stimulus modalities, and replicated the general effect in a different country, with instructions in a different language. Together, these experiments demonstrate that aesthetically moving stimuli can act as prompts for creative inspiration and provide initial evidence that the mental state of being aesthetically moved may prime a state of being creatively inspired. Our results suggest that moments of inspiration can be operationalized and that the incidence of highly inspirational episodes can be increased by priming with aesthetic stimuli.

\section{Felt Inspiration and the Creative Process}

Moments of inspiration mark a step in the creative process, and can be characterized

613 as a motivational state that marks the transition from creative ideation to actualization of an

614 idea. Inspiration is evoked by a creative idea and is an "intentional (i.e. object-focused) state,

615 which has the creative idea as its content and actualization of the idea as its aim" (Thrash et 616 al., 2010, p. 470). According to this view, inspiration is separate from ideation and could be 617 seen as a form of idea selection informed by subjective feelings of elevation, pleasure, and 618 motivation. Measuring the strength of inspirational episodes can thus serve as a measure for

619 the subjective quality of an idea (e.g. Oleynick et al., 2014; Thrash, 2020). However, it should 620 be noted that the extent to which creative ideas evoke inspiration may be modified by

621 individual approach temperament (Thrash et al., 2010) and degree to which a person sees 622 themselves as the source of an idea (Thrash, Maruskin, Moldovan, Oleynick, \& Belzak, 
624 ideation, inspiration and actualization, and inspiration could potentially occur several times

625 during successive steps. Thus, creative acts may not always entail a single strong moment of

626 inspiration. Yet, given their status as a link between cycles - their evocation follows from

627 the previous content of thought (in this case, an artwork), but also motivates the next cycle of

628 activity - inspiration is a promising window into idea generation and selection during

629 creative activity and a promising component for process models of creativity,

630 In our data, inspiration ratings were positively correlated with the amount of text

631 produced, on the single-trial level $(\mathrm{r}=0.42, \mathrm{p}<0.05)$, suggesting a relationship between felt

632 inspiration and writing fluency in this task. Similar results have been reported previously (see

633 Thrash et al., 2010). On the other hand, the participants' average amount of produced text

634 were positively correlated with their AUT-fluency scores ( $\mathrm{r}=0.39$, not significant). This is

635 not particularly surprising, since the writing task bears certain similarities with the AUT test

636 (both being time-limited text production tasks). Thus, the observed correlational structure

637 suggests a link between felt inspiration and more traditional measures of ideational fluency.

638 One possibility is that felt inspiration is a subjective response to one specific idea, and

639 interacts with the idea generation process: under many circumstances (but not in the AUT),

640 idea generation proceeds until a single idea evokes strong feelings of inspiration (e.g. a "good

641 idea”), at which point idea generation stops.

642 We defined self-reported feeling of inspiration as our main measure of interest, rather

643 than a third-party assessment of the novelty or usefulness of a specific outcome. This measure

644 reflects our interest in isolating the intensity of the subjective psychological state of creative

645 inspiration. More objective qualities of the produced texts could be included in future studies.

646 Ratings by experts and/or naïve judges (e.g. Baer \& McKool, 2009) or measures of semantic

647 distance (Heinen \& Johnson, 2018) could be compared to self-reports of the participants. In

648 fact, a similar study has already been conducted (using a scientific writing exercise)

649 suggesting a link between a writers rated inspiration and externally judged quality of the 
650

651

652

653

654

655

656

657

658

659

660

661

662

663

664

665

666

667

668

669

670

671

672

673

674

675

outcome (Thrash et al., 2010). In such a framework one could further investigate the relationship between externally rated creativity of the output with subjective aesthetic appeal for the prompt, or various objective stimulus properties. However, we would urge caution: in our study, participants had only three minutes to type starting directly after the prompt, which may not be sufficient to fully convey all their ideas in writing. This would mark a substantial confound for external ratings of the output. More generally, this study follows in the tradition of characterizing subjective psychological states independent of external measures, an approach that has been used successfully in many cognitive domains such as memory ('remember/forgotten' judgments; e.g. Brewer, 1998), visual perception (analysis of false detections and misses; e.g. Ress \& Heeger, 2003), and aesthetics (subjective aesthetic appeal; e.g. (Vessel et al., 2012). We believe this to be a fruitful future direction for understanding the psychological and neural processes underlying creative cognition.

\section{From Being Moved to Being Inspired}

Participants reported higher feelings of inspiration when writing about aesthetically moving artworks than when writing about either non-moving art or non-art prompts — as discussed above this rating likely reflects the subjective quality of their idea. While the precise temporal and logical relations of aesthetic response, idea generation, and idea selection remain to be addressed, the reported findings are consistent with our conjecture that the psychological state of being aesthetically moved may share certain features with the state of creative inspiration.

Similar to the definition of creative inspiration put forth by Thrash and Elliot (2003), moving aesthetic experiences are also evoked (often by an external object), involve feelings of transcendence beyond concrete reality (Menninghaus et al., 2015), and can motivate behavior (e.g. to repeatedly visit a gallery). Furthermore, states of strong aesthetic pleasure have been shown to require the allocation of cognitive resources (Brielmann \& Pelli, 2017), 
676 while such flexible cognitive control is also known to play a critical role in creative thinking

677 (Zabelina \& Robinson, 2010). Armstrong and Detweiler-Bedell (2008) theorized that in order

678 to experience beauty, an object (e.g. a painting) has to capture an observers attention in a way

679 that evokes imaginative processes in order to better understand the object; sustained, inwardly

680 directed imagery and prospection is also a hallmark of inspired creative thought (Benedek,

681 2018). This similarity is even alluded to in the literature on inspiration: Thrash \& Elliot

682 (2004) outline a subconstruct of inspiration that they refer to as being inspired "by," using the

683 example of appreciating the beauty of the Grand Canyon. While it is debatable whether such

684 an experience should be considered inspiration or aesthetic appeal, it is clear that these two

685 constructs overlap considerably, and may also share similarities with the construct of being

686 inspired "to" create something.

687 Several other findings indicate that being aesthetically moved and being creatively

688 inspired may engage partly overlapping neural systems. Brain imaging studies of strongly

689 moving aesthetic experiences reveal activation patterns consistent with a potential role for

690 prospection and imagery: in addition to activation of externally oriented sensory pathways

691 (e.g. the ventral visual stream), aesthetically appealing artworks also engage the DMN

692 (Vessel et al., 2012), an inwardly-focused brain network that has been shown to play a role in

693 prospection (Andrews-Hanna, Reidler, Sepulcre, Poulin, \& Buckner, 2010) and mental scene

694 construction (Axelrod, Rees, \& Bar, 2017). While the DMN is typically suppressed when a

695 person engages with external stimuli, including non-appealing artworks, aesthetically

696 appealing artworks engage the DMN, resulting in a change in the large-scale network

697 dynamics of the brain (Belfi et al., 2019; Vessel et al., 2013). Differences in DMN

698 connectivity have also been associated with trait-level creativity (Beaty et al., 2018) and there

699 is evidence that certain creative acts directly engage the DMN. For example, in a study of jazz

700 musicians, improvisation was accompanied by greater activation of cortical midline regions of 701 the DMN and less activation of lateral prefrontal regions, when compared to production of 
well-learned sequences (Limb \& Braun, 2008). A potential explanation for the observed effect of aesthetic appeal on felt inspiration is that aesthetically appealing artworks engage top-down imagery processes to a greater extent than other prompts, and that this promotes generation of more divergent and remote ideas, or improves the elaboration process.

While we posit that the states of being aesthetically moved and of feeling creatively inspired are similar, they are not the same. For example, a recent study that observed contagion of felt inspiration from the writer of a poem to a reader identified a reader's rating of pleasantness of the poem as a mediator of the contagion effect (Thrash et al., 2017), consistent with our hypothesis that the states can be linked but are not identical. In the context of the present study, it is important to note that it is unlikely that participants were implicitly rating aesthetic appeal of a prompt when they should in fact rate felt inspiration. First, participants were not asked to rate aesthetic appeal during the writing task ("We are asking you to judge the feeling of inspiration you had while starting your creative process - do not judge the prompt itself, and do not judge what you produced;" see Online Supplementary for full instructions). Furthermore, many of the stimuli (word triads in Exp. 1, novel paintings in Exp. 2) were not presented and rated for aesthetic appeal in advance; a participant would thus have had to rate aesthetic appeal of a visual memory, or recall implicit judgments during image presentation. Inspiration was rated after a three-minute writing period; the writing period and the generated idea were therefore more recent, and likely more salient in memory than the original prompt. While it is the case that aesthetic ratings and inspiration ratings were positively correlated (see Figure 4b), this correlation was significantly smaller than the correlation of repeated aesthetic ratings of the same stimuli (Hotelling's t $(419)=14.59, \mathrm{p}<$ 0.001 , two-sided) and there are numerous individual trials with artworks rated as highly moving corresponding to low ratings of felt inspiration, and vice versa (see for example Figure $3 b)$. 
For similar reasons, it is also unlikely that other judgments or feelings generated

when viewing the prompts are misattributed or transferred to the subsequent rating of felt inspiration, similar to what is known as sensation transference in the marketing literature (Cheskin, 1957). In addition to the arguments mentioned above, we found that the amount of text produced, a separate behavioral measure that was not a rating (and hence unlikely to be affected by misattribution or transference) was significantly correlated with inspiration ratings, but not with aesthetic ratings (see Figure 4b). A mediation model confirmed that even this small relationship between aesthetic ratings and text length was fully mediated by inspiration ratings (see Figure 5). These findings are more consistent with the notion that ratings of felt inspiration reflect the intervening mental process of idea generation, and are not a direct misattribution of feelings generated when viewing the prompt. We would note, however, that a potential role for other intrapsychic effects cannot be ruled out at this time. Given the potential similarity of being moved and feeling inspired, it is also conceivable that feeling inspiration when writing about a prompt might, in turn, influence subsequent ratings of aesthetic appeal. Such an effect might be caused by either increased attention to certain features of the stimulus during writing which then influence subsequent calculation of aesthetic value, or by a more specific influence of inspiration on aesthetic appeal: those stimuli that resonate with intrapsychic creative processes (causing inspiration) become more relevant to us and are subsequently experienced as more aesthetically moving. Interestingly, the correlation between inspiration ratings and post-test aesthetic judgments was significantly higher than with pre-test aesthetic judgments (Hotelling's t(419) $=-4.17, \mathrm{p}<0.001$, two-sided, see Figure 4b), and in Exp. 1 the correlation between pre- and post- aesthetic ratings in the six chosen paintings $(\mathrm{n}=150, \mathrm{r}=0.44, \mathrm{p}<0.001)$ was significantly lower than in the fourteen non-chosen ones $(n=350, r=0.61, p<0.001$; Fisher's $\mathrm{z}=-2.41, \mathrm{p}<0.05)$. Further work will be needed to determine whether the observed 
752 753

pattern was spurious, or truly reflects an effect of the writing task on subsequent aesthetic judgments.

Although our study was not powered to investigate individual differences in the relationship between being moved and being creatively inspired, we did find a mild positive relationship between PA and an observers' average text count (0.20) and a mild negative relationship between state anxiety and average text count $(-0.20)$. Future work could explore whether certain aspects of personality, mood or context might mediate or moderate the likelihood that being moved would be successfully channeled into inspiration to write.

Our findings have potential implications for other settings in which similar cycles of prompting and creative production take place, such as educational settings in which students are presented with material they should write about. Although further study would be required to see if the observed effects truly translate to educational settings, and what influence increased inspiration might have on educational success, our findings are suggestive that if students are asked to work with materials that they find aesthetically pleasing, they too might experience increased instances of creative inspiration. We would caution, though, that the setting utilized in this experiment - the open-ended creation of a short vignette in response to a prompt - might prompt a specific type of writing and may not reflect factors that are important in other types of writing. Thus, while free association creative writing is a good fit for studying the open-ended nature of ideation and inspiration, it may be less appropriate for modeling sources of inspiration and idea selection when the problem domain puts tighter constraints on the content and form.

\section{Cross-Modality Effects}

It is notable that being aesthetically moved by non-text visual stimuli (paintings) carried over and influenced inspiration in a different output modality (text), as this suggests that the underlying mechanism is at least partially independent of modality. With the 
exception of the word triads in Exp. 1, the prompts in these experiments were all visual artworks, while the requested output was text. We chose this output modality expecting that a non-artist population of participants would be most comfortable with writing as compared to any other type of creative output such as drawing, painting, singing, movement etc. Future work might specifically focus on potential modality-specific effects of prompt and output modality, such as whether a visual stimulus is better for inducing inspiration for visual output than for text. Yet our data shows that simply matching prompt and output modalities is not likely to induce higher inspiration ratings: in Exp. 1, the three-word prompts led to significantly lower ratings of felt inspiration than appealing visual artworks. We note that

787 since aesthetic ratings were not collected for the word triads and only aesthetically appealing 788 paintings were selected for the writing task, the effects of aesthetic appeal and stimulus modality in Exp. 1 cannot be statistically separated. Yet when considered alongside the results of Exp. 2, it seems more likely that aesthetic appeal was the driving factor for whether a 791 prompt led to higher felt inspiration. On the other hand, the fact that AUT fluency scores interacted with prompt category in one experiment but not in the other may speak for a modulating effect of prompt modality. In Exp. 1 (text vs. visual artworks) the effect of prompt type on felt inspiration was stronger 795 for people with lower AUT scores (a commonly used measure of trait creativity). However, 796 this effect was not seen in Exp. 2 (visual artworks only). One possibility is that the interaction 797 observed in Exp. 1 was spurious. Another possibility is that the word triads were indeed 798 particularly bad prompts for people who scored low on the AUT, and that this deficit was 799 eliminated in Exp. 2 by the use of image prompts. Given that AUT-fluency scores are a 800 measure of divergent thinking in response to word prompts, it is sensible to expect that low801 scoring participants may also perform worse at creative writing prompted by non-associated 802 words. Further research is needed to better understand the nature and source of such an interaction. 
Finally, it is important to emphasize that the results of Exp. 2 rule out a number of alternative explanations for the observed effects of prompt type on ratings of felt inspiration that could be raised in Exp. 1. The visual artworks differed from the three-word triads in many other aspects than just their aesthetic properties. Artworks contain a variety of low- and

808 high- level visual features such as color and shape that were absent in the word triads, and 809 while both artworks and words may convey semantic information, most of the artworks were

810 more semantically rich than the word lists. These factors could potentially affect the

811 performance in creative tasks directly - for example, color has been proposed to influence

812 creativity in an anagram solving task (see Mehta \& Zhu, 2009; but also Steele, 2014 who

813 failed to replicate the findings) - or might affect inspiration indirectly by causing differences

814 in other potentially relevant psychological states such as arousal. However, the persistence of 815 the effect in Exp. 2, in which a homogenous stimulus set composed only of artworks was 816 used, largely rules out such potential confounds. In addition, participants showed very low 817 agreement in which paintings they found aesthetically moving, meaning that almost all 818 paintings appeared as both moving and non-moving prompts in Exp. 2 (for different 819 participants. This suggests that neither the aesthetic ratings nor the inspiration ratings were 820 primarily driven by differences in objective stimulus properties such as the presence of 821 specific colors (see also Vessel et al., 2018).

\section{Conclusion}

We show that aesthetic appeal represents a potential mechanism to boost subjectively

824 felt inspiration in a creative writing task. While the correlation between felt inspiration and externally judged creativity or utility of an outcome needs further investigation, such a

826 behavioral intervention has high potential for both scientific and educational settings. For

827 education, one could develop targeted methods to elicit inspiring moments by interaction with 828 aesthetic material that include individual choice of what materials to engage with. Likewise, the ability to increase the incidence of inspiring moments and creative states can be valuable 
830 for increasing statistical power in empirical studies of creativity. Furthermore, the paradigm

831 presented here also allows for temporal isolation of moments of creative inspiration, a

832 necessary step for examination using the tools of cognitive neuroscience. We believe that this

833 work improves our understanding of the elevated mental states of creative inspiration and

834 aesthetic appreciation, and will aid the development of better process models of creative 835 thought.

\section{Additional Information}

837 Acknowledgements

838 The work was supported by the New York University, and the Max Planck Society. The

839 authors thank Şeyma Turk and Katherine Markowski for assisting with data collection,

840 Christine Knoop and Vanessa Kegel for feedback on German translations, David Poeppel for

841 comments on a previous draft, and Gabrielle Starr. The painting depicted in Figure 1 is a

842 digital copy of Head of a Damned Soul from Dante's "Inferno" (1770/78) by Henry Fuseli in

843 the collection of the Art Institute of Chicago, made available under Creative Commons Zero

844 (CC0) designation.

\section{Code and Data availability}

846 The manuscript was uploaded on a preprint server - https://psyarxiv.com/rdsbv. The full 847 dataset used for analysis, as well as R and MATLAB scripts replicating the results, and 848 MATLAB scripts used in data collection were made available in a public online repository 849 https://osf.io/53w6b/ 


\section{References}

851

852

853

854

855

856

857

858

859

860

861

862

863

864

865

866

867

868

869

870

871

872

873

874

875

Amabile, T. M. (1985). Motivation and creativity: Effects of motivational orientation on creative writers. Journal of Personality and Social Psychology, 48(2), 393-399. doi: $10.1037 / 0022-3514.48 .2 .393$

Andrews-Hanna, J. R., Reidler, J. S., Sepulcre, J., Poulin, R., \& Buckner, R. L. (2010). Functional-anatomic fractionation of the brain's default network. Neuron, 65(4), 550562. doi: 10.1016/j.neuron.2010.02.005

Armstrong, T., \& Detweiler-Bedell, B. (2008). Beauty as an emotion: The exhilarating prospect of mastering a challenging world. Review of General Psychology, 12(4), 305-329. doi: 10.1037/a0012558

Axelrod, V., Rees, G., \& Bar, M. (2017). The default network and the combination of cognitive processes that mediate self-generated thought. Nature Human Behaviour, l(12), 896-910. doi: 10.1038/s41562-017-0244-9

Baer, J. (1994). Divergent thinking is not a general trait: A multidomain training experiment. Creativity Research Journal, 7(1), 35-46. doi: 10.1080/10400419409534507

Baer, J., \& McKool, S. S. (2009). Assessing creativity using the consensual assessment technique. In C. Schreiner (Ed.), Handbook of Research on Assessment Technologies, Methods, and Applications in Higher Education (pp. 65-77). Hershey, PA: IGI Global. doi: 10.4018/978-1-60566-667-9.ch004

Bakdash, J. Z., \& Marusich, L. R. (2017). Repeated Measures Correlation. Frontiers in Psychology, 8. doi: 10.3389/fpsyg.2017.00456

Bates, D., Mächler, M., Bolker, B., \& Walker, S. (2015). Fitting linear mixed-effects models using lme4. Journal of Statistical Software, 67(1). doi: 10.18637/jss.v067.i01

Batey, M., \& Furnham, A. (2006). Creativity, intelligence, and personality: A critical review of the scattered literature. Genetic, Social, and General Psychology Monographs, 132(4), 355-429. doi: 10.3200/MONO.132.4.355-430 
876 Beaty, R. E., Kenett, Y. N., Christensen, A. P., Rosenberg, M. D., Benedek, M., Chen, Q., ...

877

878

879

880

881

882

883

884

885

886

887

888

889

890

891

892

893

894

895

896

897

898

899

900 Silvia, P. J. (2018). Robust prediction of individual creative ability from brain functional connectivity. Proceedings of the National Academy of Sciences, 115(5), 1087-1092. doi: 10.1073/pnas.1713532115

Belfi, A. M., Vessel, E. A., Brielmann, A., Isik, A. I., Chatterjee, A., Leder, H., ... Starr, G. G. (2019). Dynamics of aesthetic experience are reflected in the default-mode network. NeuroImage, 188, 584-597. doi: 10.1016/j.neuroimage.2018.12.017

Benedek, M. (2018). Internally directed attention in creative cognition. In R. E. Jung \& O. Vartanian (Eds.), The Cambridge Handbook of the Neuroscience of Creativity (1st ed., pp. 180-194). Cambridge, UK: Cambridge University Press. doi: $10.1017 / 9781316556238.011$

Benedek, M., Könen, T., \& Neubauer, A. C. (2012). Associative abilities underlying creativity. Psychology of Aesthetics, Creativity, and the Arts, 6(3), 273-281. doi: $10.1037 / \mathrm{a} 0027059$

Boccia, M., Piccardi, L., Palermo, L., Nori, R., \& Palmiero, M. (2015). Where do bright ideas occur in our brain? Meta-analytic evidence from neuroimaging studies of domainspecific creativity. Frontiers in Psychology, 6. doi: 10.3389/fpsyg.2015.01195

Bolte, A., Goschke, T., \& Kuhl, J. (2003). Emotion and intuition: Effects of positive and negative mood on implicit judgments of semantic coherence. Psychological Science, 14(5), 416-421. doi: 10.1111/1467-9280.01456

Boot, N., Nevicka, B., \& Baas, M. (2017). Creativity in ADHD: Goal-directed motivation and domain specificity. Journal of Attention Disorders, 108705471772735. doi: $10.1177 / 1087054717727352$

Bowers, K. S., Regehr, G., Balthazard, C., \& Parker, K. (1990). Intuition in the context of discovery. Cognitive Psychology, 22, 72-110. doi: 10.1016/0010-0285(90)90004-N 
901

902

903

904

905

906

907

908

909

910

911

912

913

914

915

916

917

918

919

920

921

922

923

924

925

926

Brainard, D. H. (1997). The psychophysics toolbox. Spatial Vision, 10(4), 433-436. doi: $10.1163 / 156856897 X 00357$

Brewer, J. B., Zhao, Z., Desmond, J. E., Glover, G. H., \& Gabrieli, J. D. E. (1998). Making memories: Brain activity that predicts how well visual experience will be remembered. Science, 281(5380), 1185-1187. doi: 10.1126/science.281.5380.1185

Brielmann, A. A., \& Pelli, D. G. (2017). Beauty requires thought. Current Biology, 27(10), 1506-1513.e3. doi: 10.1016/j.cub.2017.04.018

Carson, S. H., Peterson, J. B., \& Higgins, D. M. (2005). Reliability, validity, and factor structure of the creative achievement questionnaire. Creativity Research Journal, 17(1), 37-50. doi: 10.1207/s15326934crj1701_4

Cheskin, L. (1957). How to predict what people will buy. New York: Liveright Pub. Corp.

Coupé, C., Oh, Y., Dediu, D., \& Pellegrino, F. (2019). Different languages, similar encoding efficiency: Comparable information rates across the human communicative niche. Science Advances, 5(9), eaaw2594. doi: 10.1126/sciadv.aaw2594

Cousijn, J., Zanolie, K., Munsters, R. J. M., Kleibeuker, S. W., \& Crone, E. A. (2014). The relation between resting state connectivity and creativity in adolescents before and after training. PloS One, 9(9), e105780. doi: 10.1371/journal.pone.0105780

Diedenhofen, B. (2016). R package “cocor”: Comparing correlations (Version 1.1-3). Retrieved from http://comparingcorrelations.org/

Dietrich, A., \& Kanso, R. (2010). A review of EEG, ERP, and neuroimaging studies of creativity and insight. Psychological Bulletin, 136(5), 822-848. doi: $10.1037 / \mathrm{a} 0019749$

Doyle, C. L. (1998). The writer tells: The creative process in the writing of literary fiction. Creativity Research Journal, 11(1), 29-37. doi: 10.1207/s15326934crj1101_4

Eysenck, H. J. (1995). Genius: The natural history of creativity (Reprinted). Cambridge, UK: Cambridge Univ. Press. 
Faul, F., Erdfelder, E., Lang, A.-G., \& Buchner, A. (2007). G*Power 3: A flexible statistical power analysis program for the social, behavioral, and biomedical sciences. Behavior Research Methods, 39(2), 175-191. doi: 10.3758/BF03193146

Friedman, R. S., \& Forster, J. (2001). The effects of promotion and prevention cues on creativity. Journal of Personality and Social Psychology, 81(6), 1001-1013. doi: $10.1037 / 0022-3514.81 .6 .1001$

Gerger, G., Pelowski, M., \& Leder, H. (2018). Empathy, Einfühlung, and aesthetic experience: The effect of emotion contagion on appreciation of representational and abstract art using fEMG and SCR. Cognitive Processing, 19(2), 147-165. doi: $10.1007 / \mathrm{s} 10339-017-0800-2$

Goldstein, A. (1980). Thrills in response to music and other stimuli. Physiological Psychology, 8(1), 126-129. doi: 10.3758/BF03326460

Gonen-Yaacovi, G., de Souza, L. C., Levy, R., Urbanski, M., Josse, G., \& Volle, E. (2013). Rostral and caudal prefrontal contribution to creativity: A meta-analysis of functional imaging data. Frontiers in Human Neuroscience, 7. doi: 10.3389/fnhum.2013.00465

Grös, D. F., Antony, M. M., Simms, L. J., \& McCabe, R. E. (2007). Psychometric properties of the State-Trait Inventory for Cognitive and Somatic Anxiety (STICSA): Comparison to the State-Trait Anxiety Inventory (STAI). Psychological Assessment, 19(4), 369-381. doi: 10.1037/1040-3590.19.4.369

Guilford, J. P. (1950). Creativity. American Psychologist, 5(9), 444-454. doi:

$$
10.1037 / \mathrm{h} 0063487
$$

Guilford, J. P., Christensen, P. R., Merrifield, P. R., \& Wilson, R. C. (1978). Alternate uses: Manual of instructions and interpretation. Orange, CA: Sheridan Psychological Services.

Harrell, F. E. (n.d.). R package "Hmisc”: Harrell miscellaneous. Retrieved from http://biostat.mc.vanderbilt.edu/Hmisc 
953

Heinen, D. J. P., \& Johnson, D. R. (2018). Semantic distance: An automated measure of 954 955 956 957 958 959 960 creativity that is novel and appropriate. Psychology of Aesthetics, Creativity, and the Arts, 12(2), 144-156. doi: 10.1037/aca0000125

Howard-Jones, P. A., Blakemore, S.-J., Samuel, E. A., Summers, I. R., \& Claxton, G. (2005). Semantic divergence and creative story generation: An fMRI investigation. Cognitive Brain Research, 25(1), 240-250. doi: 10.1016/j.cogbrainres.2005.05.013

Ives, C. F., \& Metropolitan Museum of Art. (1974). The Great Wave: The Influence of Japanese Woodcuts on French Prints. New York, N.Y.: Metropolitan Museum of Art. Retrieved from https://books.google.de/books?id=JYgeaV1w9SIC

Jauk, E. (2019). A bio-psycho-behavioral model of creativity. Current Opinion in Behavioral Sciences, 27, 1-6. doi: 10.1016/j.cobeha.2018.08.012

Jauk, E., Benedek, M., \& Neubauer, A. C. (2014). The road to creative achievement: A latent variable model of ability and personality predictors: The road to creative achievement. European Journal of Personality, 28(1), 95-105. doi: 10.1002/per.1941

Julian, L. J. (2011). Measures of anxiety: State-Trait Anxiety Inventory (STAI), Beck Anxiety Inventory (BAI), and Hospital Anxiety and Depression Scale-Anxiety (HADS-A). Arthritis Care \& Research, 63(S11), S467-S472. doi: 10.1002/acr.20561

Kaufman, J. C., \& Beghetto, R. A. (2009). Beyond Big and Little: The Four C Model of Creativity. Review of General Psychology, 13(1), 1-12. doi: 10.1037/a0013688

Kuznetsova, A., Brockhoff, P. B., \& Christensen, R. H. B. (2017). lmerTest package: Tests in linear mixed effects models. Journal of Statistical Software, 82(13). doi: 10.18637/jss.v082.i13

Limb, C. J., \& Braun, A. R. (2008). Neural substrates of spontaneous musical performance: An fMRI study of jazz improvisation. PLoS ONE, 3(2), e1679. doi: 10.1371/journal.pone.0001679 
Lubart, T. I. (2001). Models of the Creative Process: Past, Present and Future. Creativity Research Journal, 13(3-4), 295-308. doi: 10.1207/S15326934CRJ1334_07

Martindale, C., Hines, D., Mitchell, L., \& Covello, E. (1984). EEG alpha asymmetry and creativity. Personality and Individual Differences, 5(1), 77-86. doi: 10.1016/01918869(84)90140-5

Mehta, R., \& Zhu, R. (2009). Blue or Red? Exploring the Effect of Color on Cognitive Task Performances. Science, 323(5918), 1226-1229. doi: 10.1126/science.1169144

Menninghaus, W., Wagner, V., Hanich, J., Wassiliwizky, E., Kuehnast, M., \& Jacobsen, T. (2015). Towards a psychological construct of being moved. PLOS ONE, 10(6), e0128451. doi: 10.1371/journal.pone.0128451

Menninghaus, W., Wagner, V., Wassiliwizky, E., Schindler, I., Hanich, J., Jacobsen, T., \& Koelsch, S. (2019). What are aesthetic emotions? Psychological Review, 126(2), 171195. doi: $10.1037 /$ rev0000135

Okada, T., \& Ishibashi, K. (2017). Imitation, Inspiration, and Creation: Cognitive Process of Creative Drawing by Copying Others' Artworks. Cognitive Science, 41(7), 1804 1837. doi: $10.1111 / \operatorname{cogs} .12442$

Oleynick, V. C., Thrash, T. M., LeFew, M. C., Moldovan, E. G., \& Kieffaber, P. D. (2014). The scientific study of inspiration in the creative process: Challenges and opportunities. Frontiers in Human Neuroscience, 8. doi: 10.3389/fnhum.2014.00436

R Core Team. (2018). R: A language and environment for statistical computing. Vienna, Austria: R Foundation for Statistical Computing. Retrieved from https://www.Rproject.org/

Ress, D., \& Heeger, D. J. (2003). Neuronal correlates of perception in early visual cortex. Nature Neuroscience, 6(4), 414-420. doi: 10.1038/nn1024

Runco, M. A., \& Acar, S. (2012). Divergent thinking as an indicator of creative potential. Creativity Research Journal, 24(1), 66-75. doi: 10.1080/10400419.2012.652929 
1004 Santarnecchi, E., Emmendorfer, A., \& Pascual-Leone, A. (2017). Dissecting the parieto-

1005

1006

1007

1008

1009

1010

1011

1012

1013

1014

1015

1016

1017

1018

1019

1020

1021

1022

1023

1024

1025

1026

1027 frontal correlates of fluid intelligence: A comprehensive ALE meta-analysis study. Intelligence, 63, 9-28. doi: 10.1016/j.intell.2017.04.008

Schindler, I., Hosoya, G., Menninghaus, W., Beermann, U., Wagner, V., Eid, M., \& Scherer, K. R. (2017). Measuring aesthetic emotions: A review of the literature and a new assessment tool. PLOS ONE, 12(6), e0178899. doi: 10.1371/journal.pone.0178899

Schlotz, W., Wallot, S., Omigie, D., Masucci, M. D., Hoelzmann, S. C., \& Vessel, E. A. (2020). The Aesthetic Responsiveness Assessment (AReA): A screening tool to assess individual differences in responsiveness to art in English and German. Psychology of Aesthetics, Creativity, and the Arts. doi: 10.1037/aca0000348

Scott, G., Leritz, L. E., \& Mumford, M. D. (2004a). The effectiveness of creativity training: A quantitative review. Creativity Research Journal, 16(4), 361-388. doi:

\section{$10.1080 / 10400410409534549$}

Scott, G., Leritz, L. E., \& Mumford, M. D. (2004b). Types of creativity training: Approaches and their effectiveness. The Journal of Creative Behavior, 38(3), 149-179. doi: 10.1002/j.2162-6057.2004.tb01238.x

Silvia, P. J. (2009). Looking past pleasure: Anger, confusion, disgust, pride, surprise, and other unusual aesthetic emotions. Psychology of Aesthetics, Creativity, and the Arts, 3(1), 48-51. doi: 10.1037/a0014632

Snaith, R. P., Hamilton, M., Morley, S., Humayan, A., Hargreaves, D., \& Trigwell, P. (1995). A scale for the assessment of hedonic tone the Snaith-Hamilton Pleasure Scale. British Journal of Psychiatry, 167(1), 99-103. doi: 10.1192/bjp.167.1.99

Spielberger, C. D. (1983). Manual for the State-Trait Anxiety Inventory (Form Y). Palo Alto CA: Consulting Psychologists Press. 
1028 Steele, K. M. (2014). Failure to replicate the Mehta and Zhu (2009) color-priming effect on

1029

1030

1031

1032

1033

1034

1035

1036

1037

1038

1039

1040

1041

1042

1043

1044

1045

1046

1047

1048

1049

1050

1051

1052

1053 anagram solution times. Psychonomic Bulletin \& Review, 21(3), 771-776. doi: 10.3758/s13423-013-0548-3

Sternberg, R. J., \& Davidson, J. E. (Eds.). (1995). The nature of insight. Cambridge, MA: MIT Press.

Thrash, T. M. (2020). The creation and curation of all things worthy: Inspiration as vital force in persons and cultures. In Advances in Motivation Science. Elsevier. doi: 10.1016/bs.adms.2020.01.002

Thrash, T. M., \& Elliot, A. J. (2003). Inspiration as a psychological construct. Journal of Personality and Social Psychology, 84(4), 871-889. doi: 10.1037/0022-3514.84.4.871

Thrash, T. M., Maruskin, L. A., Cassidy, S. E., Fryer, J. W., \& Ryan, R. M. (2010). Mediating between the muse and the masses: Inspiration and the actualization of creative ideas. Journal of Personality and Social Psychology, 98(3), 469-487. doi: 10.1037/a0017907

Thrash, T. M., Maruskin, L. A., Moldovan, E. G., Oleynick, V. C., \& Belzak, W. C. (2017). Writer-reader contagion of inspiration and related states: Conditional process analyses within a cross-classified writer $\times$ reader framework. Journal of Personality and Social Psychology, 113(3), 466-491. doi: 10.1037/pspp0000094

Tingley, D., Yamamoto, T., Hirose, K., Keele, L., \& Imai, K. (2014). mediation: R Package for Causal Mediation Analysis. Journal of Statistical Software, 59(5). doi: 10.18637/jss.v059.i05

Tinio, P. P. L. (2013). From artistic creation to aesthetic reception: The mirror model of art. Psychology of Aesthetics, Creativity, and the Arts, 7(3), 265-275. doi: $10.1037 / \mathrm{a} 0030872$

Torrance, E. P. (1968). A longitudinal examination of the fourth grade slump in creativity. Gifted Child Quarterly, 12(4), 195-199. doi: 10.1177/001698626801200401 
1054 Vessel, E. A., Maurer, N., Denker, A. H., \& Starr, G. G. (2018). Stronger shared taste for

1055

1056

1057

1058

1059

1060

1061

1062

1063

1064

1065

1066

1067

1068

1069

1070

1071

1072

1073

1074

1075

1076

1077

1078

1079 natural aesthetic domains than for artifacts of human culture. Cognition, 179, 121131. doi: 10.1016/j.cognition.2018.06.009

Vessel, E. A., Starr, G. G., \& Rubin, N. (2012). The brain on art: Intense aesthetic experience activates the default mode network. Frontiers in Human Neuroscience, 6. doi: 10.3389/fnhum.2012.00066

Vessel, E. A., Starr, G. G., \& Rubin, N. (2013). Art reaches within: Aesthetic experience, the self and the default mode network. Frontiers in Neuroscience, 7. doi: $10.3389 /$ fnins .2013 .00258

Wallas, G. (1926). The art of thought. London, UK: Jonathan Cape.

Wassiliwizky, E., Wagner, V., Jacobsen, T., \& Menninghaus, W. (2015). Art-elicited chills indicate states of being moved. Psychology of Aesthetics, Creativity, and the Arts, 9(4), 405-416. doi: 10.1037/aca0000023

Watson, D., \& Clark, L. A. (1999). The PANAS-X: Manual for the positive and negative affect schedule - expanded form. doi: 10.17077/48vt-m4t2

Wei, T., \& Simko, V. (2017). R package "corrplot": Visualization of a correlation matrix (Version 0.84). Retrieved from https://github.com/taiyun/corrplot

Zabelina, D. L., \& Robinson, M. D. (2010). Creativity as flexible cognitive control. Psychology of Aesthetics, Creativity, and the Arts, 4(3), 136-143. doi: $10.1037 / \mathrm{a} 0017379$

Zenasni, F., \& Lubart, T. (2002). Effects of mood states on creativity. Current Psychology Letters [Online], 2002/2(8), 33-50.

Zentner, M., Grandjean, D., \& Scherer, K. R. (2008). Emotions evoked by the sound of music: Characterization, classification, and measurement. Emotion, 8(4), 494-521. doi: $10.1037 / 1528-3542.8 .4 .494$ 
1080

1081

1082

1083 Table A1

1084 List of word triads used in Experiment 1

\begin{tabular}{lll}
\hline Hard & Bang & Fountain \\
Colors & Salt & Sum \\
Poke & Lettuce & Molasses \\
\hline Trumpet & Big & Gold \\
Bathtub & Hair & Cracker \\
Grant & Scotch & Wheel
\end{tabular}

\section{Appendix A}

\section{Full list of Stimuli}

\footnotetext{
Note. Word triads taken from Bowers et al. (1990)
} 
1086 Table A2

1087 List of painting stimuli

\begin{tabular}{|c|c|c|}
\hline Title & Artist & Year \\
\hline \multicolumn{3}{|l|}{ Paintings used in Experiment 1 and 2} \\
\hline Classical Landscape & Joshua Shaw & 1830 \\
\hline Death on the Pale Horse & Benjamin West & 1796 \\
\hline \multicolumn{3}{|l|}{ Die Junge Morgenländerin (The Young Eastern } \\
\hline & Friedrich von Amerling & 1838 \\
\hline \multicolumn{3}{|l|}{ Woman) } \\
\hline \multicolumn{3}{|l|}{ Fellah Women Drawing Water (Medinet-el- } \\
\hline & Jean Leon Gerome & $19^{\text {th }}$ century \\
\hline \multicolumn{3}{|l|}{ Fayoum) } \\
\hline Girl In Glitz & Mary Pratt & 2002 \\
\hline Greek Slave & Jean Leon Gerome & 1870 \\
\hline Head of a Damned Soul from Dante's "Inferno" & Johann Heinrich Füssli & $1770-1778$ \\
\hline Lady Seated & Jules Adolphe Goupil & $19^{\text {th }}$ century \\
\hline Medusa & Alice Pice Barney & 1892 \\
\hline Niagara Falls from Table Rock & Samuel Finley Breese Morse & 1835 \\
\hline On The Beach & Alfred Pellan & 1845 \\
\hline Portrait of Madame $X$ & Giovanni Boldini & 1907 \\
\hline Prometheus Bound & Thomas Cole & $1846-1847$ \\
\hline The Beeches & Asher B. Durand & 1845 \\
\hline The Bouquet & Raimundo Madrazo y Garreta & 1870 s \\
\hline The Nightmare & Henry Fuseli & 1781 \\
\hline The Questioner of the Sphinx & Elihu Vedder & 1863 \\
\hline The Wreck & Eugene-Louis-Gabriel Isabey & 1854 \\
\hline Turning Point of Thirst & Victor Brauner & 1934 \\
\hline Watson and the Shark & John Singleton Copley & 1782 \\
\hline
\end{tabular}

Additional paintings used as novel prompts in Experiment 2 
An Ecclesiastic

Hercules as Heroic Virtue Overcoming Discord

Cloud Study
Mariano Fortuny y Marsal

Peter Paul Rubens

John Constable
1874

$1632-1633$

C. 1822

1088 Note. Painting titles and artist names taken from the Catalog of Art Museum Images Online (CAMIO) - this

1089 database was closed Dec. 2018 and cannot be accessed anymore. 
1090

1091

1092

1093

1094

1095

1096

1097

1098

1099

1100

1101

1102

1103

1104

1105

1106

1107

1108

1109

1110

1111

1112

1113

1114

\section{Appendix B}

\section{Across study comparison of responses}

We aimed to test whether there were significant differences in the participants rating behavior across the two experiments using a series of two-sided t-tests. In both Exp. 1 and Exp. 2, moving artworks were chosen by means of an aesthetic judgment pre-test, worked on in the aesthetic writing task, and rated again for aesthetic appeal in a post-test session.

Comparison of responses was hence restricted to this subset of data collected under almost identical conditions. The average aesthetic rating in the pre-test session was significantly lower in Exp. $1(\mathrm{M}=0.79,95 \%$ CI $[.774, .813])$ than in Exp. $2(\mathrm{M}=0.86,95 \%$ CI $[.838$, $.876]$; two-tailed paired $t(281.96)=-4.59, p<0.01,95 \%$ CI $[-.091,-.036]$, Cohen's $d=-0.54$, 95\% CI $[-.782,-.306])$. This is not surprising, since in Exp. 1 the top 6 highest rated paintings were selected for the writing task, while in Exp. 2 only the top 4 were taken. The average aesthetic rating in the post-hoc session, however was not significantly different in Exp. 1 (M $=0.73,95 \%$ CI $[.698, .76])$ than in Exp. $2(\mathrm{M}=0.76,95 \%$ CI $[.721, .797]$, two-tailed paired $t(266.39)=-1.19, p=0.237,95 \%$ CI $[-.078, .019]$, Cohen's $d=-0.14,95 \%$ CI [-.376, .092]). The average inspiration rating in the writing session of Exp. $1(\mathrm{M}=0.63,95 \% \mathrm{CI}$ $[.591, .675])$ was also not significantly different from Exp. 2 (M = 0.64, 95\% CI [.596, .683]; two-tailed paired $t(279.64)=-0.20, p=0.838,95 \%$ CI $[-.067, .054]$, Cohen's $d=-0.02,95 \%$ CI $[-.258, .210])$. Finally, average text count in the writing session was significantly higher in Exp. $1(\mathrm{M}=481.9,95 \%$ CI $[455.65,508.15])$ than in Exp. $2(\mathrm{M}=419.5,95 \%$ CI $[387.79$, 451.20], two-tailed paired $t(268)=3.0, p<0.01,95 \%$ CI [21.41, 103.40], Cohen's $d=0.36$, 95\% CI $[.123, .594])$. The mean difference in typed characters per trial equals roughly 5-15 words. AUT-fluency scores, however, were not significantly different across the two samples (Exp. 1: $\mathrm{M}=8.36,95 \%$ CI [7.16,9.54]; Exp. 2: $\mathrm{M}=7.65,95 \%$ CI $[6.45,8.84]$; two-tailed paired $t(55.92)=0.87, p=0.389,95 \%$ CI [-0.93, 2.36], Cohen's $d=0.22,95 \%$ CI [-.306, 
$1115.752])$. As Exp. 1 was conducted in English with fluent English speakers while Exp. 2 was

1116 conducted in German with fluent German speakers, language may have systematically

1117 influenced typing speed (as has been shown for spoken language (e.g. Coupé, Oh, Dediu, \&

1118 Pellegrino, 2019). 


\section{Appendix C}

\section{Correlation tables}

\section{Table C1}

1122 Pairwise correlation coefficients for participant-level covariates.

\begin{tabular}{|c|c|c|c|c|c|c|c|c|c|c|c|c|}
\hline Variable & $n$ & $M$ & SD & 1 & 2 & 3 & 4 & 5 & 6 & 7 & 8 & 9 \\
\hline 1. AGE & 59 & 26.98 & 8.264 & - & & & & & & & & \\
\hline 2. AUT & 59 & 7.95 & 3.192 & -0.05 & - & & & & & & & \\
\hline 3. AReA & 59 & 48.85 & 17.565 & 0.22 & 0.05 & - & & & & & & \\
\hline 4. $P A$ & 59 & 17.25 & 3.924 & 0.19 & 0.09 & 0.15 & - & & & & & \\
\hline 5. NA & 59 & 8.00 & 3.179 & 0.32 & -0.18 & 0.30 & 0.08 & - & & & & \\
\hline 6. STAIs & 59 & 37.53 & 9.573 & 0.04 & -0.04 & 0.06 & $-0.47^{*}$ & 0.42 & - & & & \\
\hline 7. STAlt & 59 & 41.31 & 11.627 & 0.04 & 0.04 & 0.27 & -0.41 & 0.24 & $0.63 *$ & - & & \\
\hline 8. SHAPS & 34 & 12.85 & 1.395 & 0.15 & 0.11 & -0.11 & 0.20 & 0.07 & -0.17 & -0.46 & - & \\
\hline 9. mTXT & 59 & 447.5 & 162.37 & -0.21 & 0.39 & 0.20 & 0.20 & -0.05 & -0.20 & 0.11 & -0.18 & - \\
\hline
\end{tabular}

1123 Note. Combined data from Exp. 1 and 2 (n=59): AGE; AUT-score; questionnaire scores (AReA; PA, NA -

1124 PANAS subscores; STAIs, STAIt - STAI subscores; SHAPS); and mean text count over all 12 trials (mTXT).

1125 a SHAPS data was only collected in Exp. 2.

$1126 * \mathrm{p}<0.05$, Holm-corrected. 


\section{Table C2}

1128 Pairwise correlation coefficients for single-trial-level responses.

\begin{tabular}{lccccccc}
\hline \multicolumn{1}{c}{ Variable } & $\mathrm{n}$ & $\mathrm{M}$ & $\mathrm{SD}$ & 1 & 2 & 3 & 4 \\
\hline 1. AE-PRE & 420 & 0.62 & 0.317 & - & & & \\
2. AE-POS & 420 & 0.59 & 0.307 & $0.78^{*}$ & - & & \\
3. INSP & 420 & 0.59 & 0.268 & $0.31^{*}$ & $0.45^{*}$ & - & \\
4. TXT & 420 & 440.0 & 183.50 & 0.06 & 0.16 & $0.42^{*}$ & -
\end{tabular}

1129 Note. Combined data from Exp. 1 and 2 ( $\mathrm{n}=420$ trials from 59 participants). Inspiration ratings (INSP); text

1130 count (TXT); and corresponding aesthetic ratings from pre- and post-tests (AE-PRE, AE-POS). Only data from

1131 trials with all four measures were included, i.e. moving paintings from Exp. 1 and moving and non-moving

1132 (familiar) paintings from Exp. 2. Correlation coefficients and $\mathrm{p}$ values obtained via repeated measures

1133 correlation (Bakdash \& Marusich, 2017).

$1134 * \mathrm{p}<0.05$, Holm-corrected. 